Check for updates

Cite this: J. Mater. Chem. B, 2019, 7,3480

Received 21st March 2019, Accepted 14th May 2019

DOI: $10.1039 / c 9 t b 00557 a$

rsc.li/materials-b

\section{Recent advances in gold nanoparticles for biomedical applications: from hybrid structures to multi-functionality}

\author{
Hyeon-Ho Jeong, (DD ${ }^{\text {ab }}$ Eunjin Choi, ${ }^{a}$ Elizabeth Ellis ${ }^{c d}$ and Tung-Chun Lee (D) *ce
}

${ }^{a}$ Max Planck Institute for Intelligent Systems, Heisenbergstr. 3, 70569 Stuttgart, Germany

${ }^{b}$ Cavendish Laboratory, University of Cambridge, J. J. Thomson Avenue, Cambridge CB3 OHE, UK

${ }^{c}$ Department of Chemistry, University College London (UCL), 20 Gordon Street, WC1H OAJ London, UK. E-mail: tungchun.lee@ucl.ac.uk

${ }^{d}$ Institute for Materials Research and Engineering (IMRE), Agency for Science Technology and Research ( $\left.A^{*} S T A R\right)$, Singapore

${ }^{e}$ Institute for Materials Discovery, University College London (UCL), UK

\section{Introduction}

Gold nanoparticles (Au NPs) are typically defined as particles of $1-100 \mathrm{~nm}$ in size, ${ }^{1}$ which is in the sub-wavelength regime of visible light. They often find applications in biology and medicine owing to their unique physiochemical properties, including their small and tailorable size, high chemical stability and biocompatibility, availability via facile liquid-phase

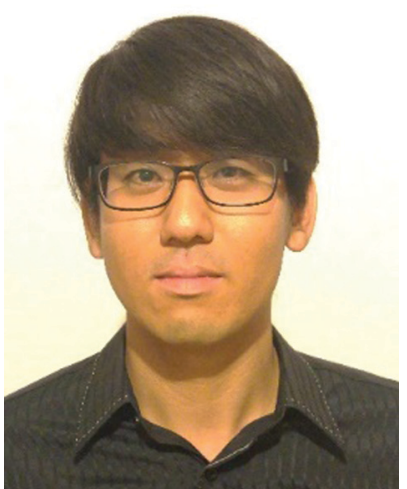

Hyeon-Ho Jeong
Dr Hyeon-Ho Jeong is a research associate at the Cavendish Laboratory, University of Cambridge. He received his BEng and MEng in electrical engineering from Dankook University, respectively, in 2010 and 2011. He then moved to Seoul National University Medical Research Centre as a research assistant for a year. In 2017, he received his $P h D$ in Material engineering from Max Planck Institute for Intelligent Systems and Swiss Federal Institute of Technology in Lausanne (EPFL), with two graduate student awards, respectively, from the American and the European Material Research Societies in 2016 and 2017. His research interests include nanomaterials, nanofabrication, and plasmonics.

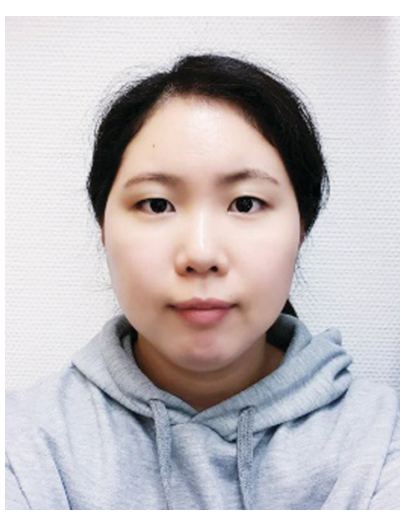

Eunjin Choi
Eunjin Choi is a PhD student at Max Planck Institute for Intelligent Systems. She received her BSc in Applied chemical engineering from Korea University of Technology and Education in 2014 and MSc in Materials science from University of Stuttgart in 2017. She is currently working on several projects under the supervision of Prof. Peer Fischer at Micro, Nano, and Molecular Systems Lab. Her research interests include acoustic fabrication, organ phantoms, tissue-like materials, and micro actuators with a focus in medical applications. 
synthesis and surface modification, and unique optical properties. $^{2,3}$

Similar to their bulk analogue, Au NPs are well-known for their chemical stability. In particular, they are generally stable against oxidation under physiological conditions (incl. variable $\mathrm{pH}$, ionic strength and temperature) without any major risk of leaching of toxic species. ${ }^{4}$ Meanwhile, the surface of Au NPs can be easily functionalised by a wide variety of ligands via thiol or amine chemistry. These two properties enable Au NPs to be utilised as an ideal nanoscale platform for drug and gene delivery amongst a range of nanocarriers. ${ }^{5}$

Nevertheless the key characteristic that distinguishes $\mathrm{Au}$ NPs from many other nanomaterials, in the biomedical context, is their unique optical properties resulting from a physical phenomenon known as localised surface plasmon resonance (LSPR), ${ }^{6}$ which is present typically in nanostructures of plasmonic materials, such as gold, silver, copper and aluminium. LSPR involves coherent oscillation and excitation of conduction-band electrons on the surface of plasmonic nanostructures, such as Au NPs, upon irradiation of light in the ultraviolet-visible-near infrared (UV-VIS-NIR) spectral region.

In the context of light-matter interaction, LSPR is typically stronger than that of small molecules because of a better match in length scale between the travel distance of localised surface plasmon and the wavelength of UV-vis-NIR light and the large polarisability of plasmonic materials at optical frequency. LSPR accompanies optical near-field enhancement that interacts with the medium surrounding a nanoparticle. ${ }^{7}$ This distinctive optical interaction affects the associated far-field spectral resonance signal and, at the same time, can generate heat locally. ${ }^{8}$ The strong interaction with light facilitates the use of Au NPs as nano-antennae for revealing optical properties of the nanoscale environment around the nanoparticles, leading to various types of sensing applications, ${ }^{9}$ as well as for nanolithography, ${ }^{10}$ photothermal therapy, ${ }^{4}$ object trapping, ${ }^{11}$ and nanoscale propulsion via thermophoretic effect. ${ }^{12}$

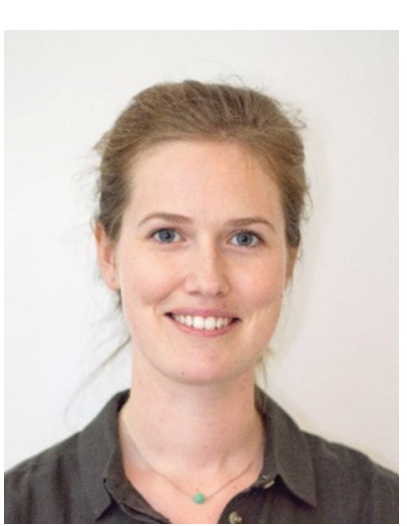

Elizabeth Ellis
Elizabeth Ellis received her MSci in Chemistry from the University of Bristol in 2014. She is currently studying for a PhD at University College London under the supervision of Dr Tung-Chun Lee, with a two-year research attachment at the Agency for Science, Technology and Research $\left(A^{*} S T A R\right)$ in Singapore, under the supervision of Dr Xian Jun Loh. Her research interests include the development of selfassembled polymeric nanostructures and hybrid nanoparticles for biomedical applications.

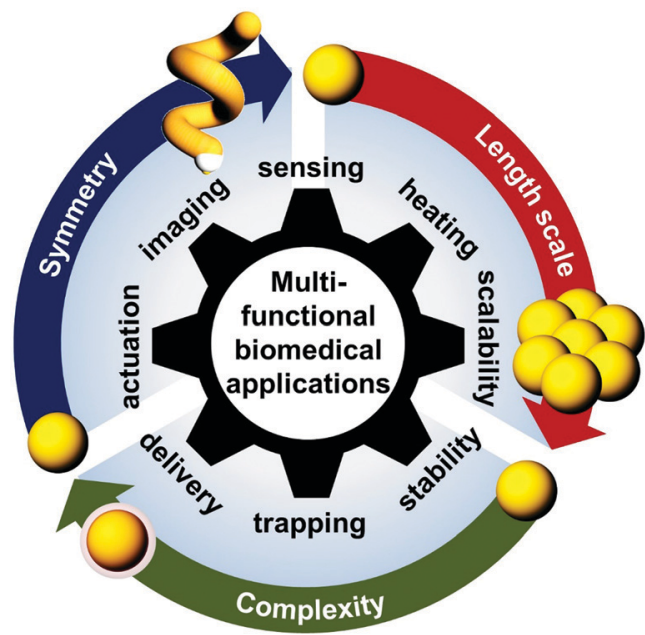

Fig. 1 Scheme showing functionalities of hybrid Au NPs achieved by engineering their structural dimensions for biomedical applications.

The conditions for the occurrence of LSPR are known to be highly sensitive to the three-dimensional (3-D) geometric parameters (e.g. size, shape and symmetry), the material composition and distribution within a NP, as well as the overall arrangement of NPs within an ensemble or array. ${ }^{13,14}$ In particular, dipolar LSPR of individual spherical Au NPs occurs in the visible region of $\sim 520-540 \mathrm{~nm}$ and can be shifted to the NIR region in the case of elongated NPs (i.e. large shape anisotropy) or assemblies of NPs with nano-spacing. As a result, optical properties of Au NPs (e.g. peak position, bandwidth and the number of resonant modes) can be engineered by designing and optimising structural and material dimensions to suit a specific biomedical application, as illustrated in Fig. 1.

In addition to tailoring the optical properties, incorporation of other functional materials onto a Au NP construct can also introduce other physiochemical properties/functionalities, including magnetism, stimuli-responsiveness, antifouling and

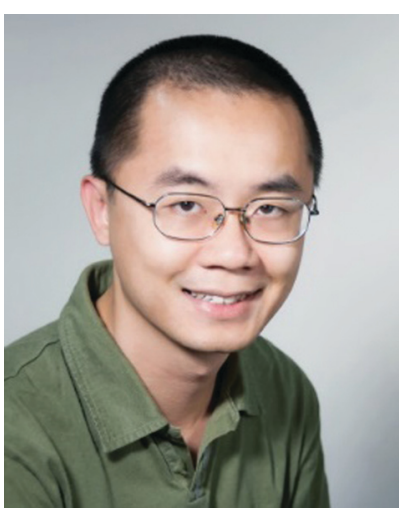

Tung-Chun Lee
Dr Tung-Chun Lee received his BSc in Chemistry from University of Hong Kong in 2005 and his PhD in Chemistry from University of Cambridge in 2012. He was a Postdoctoral Fellow at the Max Planck Institute for Intelligent Systems from 2011 to 2014 before he joined the University College London as a Lecturer and started his independent academic career. His research interests include exotic nanoparticles, nanofabrication, nanochemistry, active matter and supramolecular chemistry. Currently, he is leading an interdisciplinary group of 15 researchers exploring chemistry under nano-confinement, active propulsion at the nanoscale and metamaterial sensors. 
cell targeting..$^{15-17}$ The combination of two or more molecular ligands, polymer coatings and inorganic materials can lead to multifunctional hybrid NPs which can show superior performance in coping with complex biological environments. The facile chemistry in synthesis and functionalisation of Au NPs makes them a promising nano-scaffold for constructing hybrid NPs with multi-functionality. ${ }^{18}$ Nevertheless, producing bespoke complex shapes with programmable material distribution at the nanoscale remains a challenge because minimisation of surface energy and crystallisation forces generally leads to highly symmetric and homogeneous nanostructures. ${ }^{19}$

Recent advances in chemical synthesis and 3-D nanofabrication techniques offer new promise in the production of complex hybrid Au NPs in practical quantity and quality. These novel hybrid nanoparticles give rise to multi-functionality which opens up exciting opportunities in their biomedical applications. Unlike previous reviews on Au NPs' synthesis and biomedical applications, ${ }^{2,3,20,21}$ we here highlight recent development of complex 'hybrid' Au NPs. First, we propose a classification framework for gold nanostructures based on three fundamental structural dimensions (length scale, complexity and symmetry) for classifying and designing novel gold nanostructures. The framework can also be extended to classify other type of NPs. The second part of the review is dedicated to discussing key physiochemical properties exhibited by novel hybrid Au NPs, and their potential biomedical applications, featuring brand-new functions that are unachievable using conventional Au NPs, for instance, direct measurement of blood plasma viscosity at human haematocrit level (ca. 50\%) and active drug delivery using self-propelling nanomotors.

\section{Classification framework}

The past two decades have witnessed a boom in the development of complex hybrid Au NPs. Despite classical gold nanospheres being the primary choice in many biomedical research and applications, an increasing number of complex Au NPs have become commercially available, ranging from nanorods to nanocubes and $\mathrm{Au}-\mathrm{SiO}_{2}$ core-shell NPs, with various types of bespoke surface coating. Meanwhile novel hybrid Au nanostructures are being reported in the scientific community at an impressive rate (see Section 4 for details).

With an aim of gaining a clear overview of the highly diverse hybrid gold nanostructures, we propose a classification framework based on three fundamental structural dimensions, namely length scale, complexity and symmetry. Meanwhile gold nanostructures are grouped into three main classes, namely (1) homogeneous Au NPs, (2) heterogeneous Au NPs and (3) Au NP assemblies, according to their length scale and the material's complexity. Representative subclasses can then be organised with respect to each other according to the structural dimensions, as shown in Fig. 2.

This classification framework can aid comparison and design of nanomaterials in a systematic fashion. While many existing examples of gold nanomaterials are not featured in the

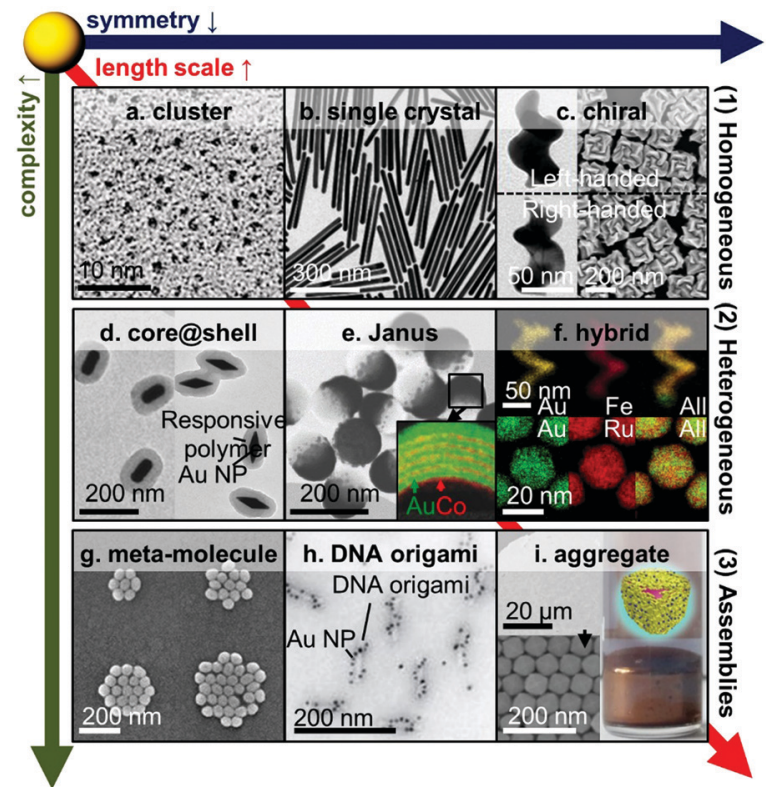

Fig. 2 Hybrid Au nanoparticles. (a) Clusters, $^{47}$ (b) single crystal nanowire, ${ }^{52}$ (c) chiral NPs, ${ }^{31,45}$ (d) core-shell NPs, ${ }^{27}$ (e) Janus NPs, ${ }^{23}$ (f) alloyed NPs, ${ }^{142,174}$ (g) plasmonic meta-molecules, ${ }^{175}$ (h) DNA-origami templated assemblies, ${ }^{25}$ (i) large-scale assemblies of NPs. ${ }^{99,176}$ Adapted by permission from the corresponding references. Copyright American Chemical Society, Springer Nature, Wiley-VCH.

scheme owing to limitation of space, other nanostructures can be readily inserted into the framework by benchmarking against examples in the scheme based on the fundamental structural parameters. For instance, Au nanostars are low symmetry and made of pure gold, ${ }^{22}$ so they can be positioned between rods (Fig. 2b) and helices (Fig. 2c). Detailed discussions on the three main classes can be found in Section 2.4.

The relevance of the structural dimensions to the potential biomedical applications of the nanomaterials and the corresponding consideration about nanomaterials design will be briefly discussed below.

\subsection{Length scale}

The length scale of an individual gold nano-object typically lies in the range of 1-100 nm, and that of composite and selfassembled nanostructures generally spans a much wider range up to the micron regime. While length scale in this context refers to the overall size of a nano-object, it is noted that, in the case of hierarchical nanostructures, a nano-object can have a feature size much smaller than the overall length scale of the NP. For instance, the Janus Co-Au multilayer coated NPs (overall size $\sim 100 \mathrm{~nm}$ ) consist of alternate layers of gold $(6 \mathrm{~nm})$ and cobalt $(1 \mathrm{~nm})$ as shown in Fig. $2 \mathrm{e}^{23}$

The overall size of a nanoparticle will have major effects on cellular interactions and in vivo pharmacokinetics, including cellular uptake, blood circulation half-life, biodistribution and tumour permeability. ${ }^{24}$ For prolonged blood circulation halflife, the NPs should be within the size window of 10-150 nm. In cases where rapid renal clearance is desirable, the NPs should be smaller than $10 \mathrm{~nm}$, which is the effective cut-off size of the 
renal filtration barrier. Within the size window, larger nanoparticles tend to have lower colloidal stability owing to the large van der Waals interactions between them. To screen the attractive interactions and therefore enhance the colloidal stability in aqueous media, coating the surface of nanoparticles with appropriate materials (e.g. hydrophilic small molecules for small $\mathrm{Au} \mathrm{NPs,} \mathrm{highly} \mathrm{hydrophilic} \mathrm{polymers} \mathrm{or} \mathrm{inorganic}$ materials for large Au NPs) is generally required for most in vitro and in vivo applications.

\subsection{Material complexity}

Complexity here refers to the number of types of materials and molecules within a given nanostructure and the degree of order amongst individual components. Simple systems typically consist of homogeneous Au NPs stabilised by a single type of ligand, whereas complex systems can involve $>200$ molecularly precise components as found in DNA-origami templated Au NP assemblies (Fig. 2h). ${ }^{25}$

The purpose of incorporating other materials and molecular components in a gold nanostructure is to address basic design requirements of the service life cycle of a given biomedical application, as well as to introduce multiple functionalities within a single nanostructure.

Service life cycle design is particularly crucial for in vivo applications. In addition to considerations on NP size discussed in Section 2.1, a number of requirements, many of them nonnegotiable, have to be fulfilled when designing hybrid Au NPs. For instance, the NPs have to be water-soluble, colloidally stable and biocompatible. On top of that, the NPs have to perform the predefined function specifically and efficiently. Since biological systems are highly complex in nature, the ideal NPs will need to cope with the complex environment while being transported to the target region and trigger a specific task on-demand. This can only be achieved by incorporating and optimising multiple functionalities from various functional molecular, polymeric and material components on the NP platform.

Furthermore, entirely new properties and functions can arise by carefully combining functional materials. For instance, forming the Pt layer surrounding Au NPs permits detection of p24, one of the earliest and most conserved biomarkers of human immunodeficiency viruses (HIV), via lateral flow immunoassay in 20 min with $10^{4}-10^{5}$ of the molecular dynamic range (roughly two-fold larger than the commercial products). ${ }^{26}$ This is not possible with either material alone.

The production of hybrid Au NPs can be achieved by various approaches. The simplest route is via a linear series of stepwise reactions, such as the formation of core-shell nanostructures by growing a layer of material around the Au NP core (Fig. 2d). ${ }^{27,28}$ The shell layer can then be further functionalised by another material or ligand. However, the level of complexity and structural diversity can be limited by the yield of each step in the series. This issue can be overcome by using either a convergent approach or a divergent approach, both of which have been commonly used in synthesis of complex organic molecules.

The convergent approach involves the synthesis of individual components, followed by the combination of all individual components to form the final product. This is an effective way to increase the degree of complexity of a nanostructure without compromising the synthetic yield. Supramolecular self-assembly is often employed in the final step of combining individual components because of its mild reaction conditions and the tendency to reach the desired state of thermodynamic equilibrium via dynamic exchange of supramolecular bonds. The latter is particularly important to the successful formation of highly complex structures which is prone to inhibition by kinetically trapped states. For instance, the synthesis of DNA-origami templated assemblies (Fig. 2h) was typically performed via the preparation of functional Au NPs and the DNA-origami scaffold, followed by the combination step via aqueous self-assembly. ${ }^{25}$

The divergent approach involves the initial synthesis of an intermediate which can subsequently be converted into a wide range of nanostructures. This approach can efficiently produce a library of chemically or structurally related nanostructures. Examples include structurally similar Au nanocages from $\mathrm{Ag}$ nanocubes, ${ }^{29}$ as well as Au NPs coated by a mixed self-assembled monolayer with various ligand composition from surfactantstabilised Au NPs. ${ }^{30}$

On top of the above approaches, the conflict between yield and complexity of hybrid Au NP synthesis can be tackled by fundamentally developing fabrication techniques with (close to) quantitative yield. For instance, nanoscale glancing angle deposition (nano-GLAD) is a novel 3-D nanofabrication technique that allows direct growth of hybrid Au NPs (and other nanostructures) in a parallel manner, producing Au NPs with high degree of complexity of a practical amount. ${ }^{31,32}$ In contrast, dip-pen lithography, which is a nanoscale printing technique, can be used to fabricate increasingly complex hybrid NPs, but the throughputs remain insufficient for the practical use. ${ }^{33,34}$

\subsection{Symmetry and chirality}

Symmetry of a discreet nano-object can be classified based on symmetry point groups ( $K$ for a perfect sphere, $D_{\infty \mathrm{h}}$ for a rod, $C_{\infty \mathrm{v}}$ for a Janus particle, $C_{2}$ for a helix and $C_{1}$ if there does not exist any symmetry element other than $C_{1}$ which is equivalent to the identity element $E$ ). In general, a nanostructure is referred as less symmetric if it belongs to a point group with fewer symmetry elements.

For a given symmetry, the aspect ratio of a nanostructure describes the ratio between the length of the structure along the principle axis (i.e. that of highest rotational symmetry) to the width perpendicular to the principle axis. The aspect ratio is reflected as the shape factor $\chi$ in the Mie solution to the Maxwell equation, where the conditions of a particular LSPR mode are given as $\varepsilon_{\mathrm{r}}=-\chi \varepsilon_{\mathrm{m}}{ }^{7}$ Here, $\varepsilon_{\mathrm{r}}$ is the real dielectric constant of metal NP and $\varepsilon_{\mathrm{m}}$ is a refractive index of the surrounding medium. For sphere, $\chi=2$ while for $\operatorname{rod}>2$. It is noted that LSPR can be red-shifted to the NIR range with increasing shape factor (i.e. aspect ratio) of the nanostructure, which can be useful for in vivo applications due to the long penetration depth of NIR radiation through skin and tissue. Moreover, sensitivity of a plasmonic sensor also increases with the shape factor and thus the resonance wavelength of the LSPR 
mode (Section 3.1.1); meanwhile background fluorescence generally decreases with increasing excitation wavelength in surfaceenhanced Raman scattering measurements (Section 3.1.2). Both of these cases benefit from shifting the LSPR to the NIR regime. Nevertheless, the use of NIR light accompanies a reduction in spatial resolution of the sensing probe, as well as expensive and complex optical settings. Therefore, optimisation based on casespecific design constraints is often required in practical scenarios.

Decreasing the symmetry of a nanostructure can give rise to interesting optical effects. In particular, a nanostructure is classified as chiral if it falls into any of the low symmetry point groups without a plane of symmetry $\left(C_{1}, C_{2}, C_{3}, D_{2}, D_{3}\right)$. As the shape of chiral Au NPs (Fig. 2c) cannot be superimposed onto its mirror image by simple rotation or translation, they exhibit chiroptical effects near the frequency of the LSPR. Despite the presence of analogous chiroptical effects in pure enantiomers of chiral small molecules, chiroptical effects originated from LSPR are typically orders of magnitude more pronounced because of their strong light-matter interaction. As a result, chiral $\mathrm{Au}$ NPs find potential applications in backgroundfree optical sensing in optically dense bio-media (Sections 3.1 and 3.3), as well as environmental toxins in complex sample mixtures such as soil.

Another unique property of chiral NPs is the strong coupling between rotational and translational motion in fluid media. For instance, when a helical NP is rotating unidirectionally around its long-axis, it can produce linear propulsion force and translate in a single direction through a fluid, despite the presence of strong Brownian forces at the nanoscale. Furthermore, this type of rotation breaks the time-reversal symmetry of the system, and therefore enables the helical NP to propel through media at the low Reynolds number regime, which is a scenario when viscous forces dominate over inertia and is common among micro- and nanoscale systems. This propulsion strategy has been used by microorganisms, such as E. coli (Escherichia coli), found in nature for millions of years, but has been demonstrated in artificial micro-/nanosystems only in recent years. ${ }^{35}$ The exciting possibilities of generating and controlling propulsion forces down to the nanoscale promise a wide range of novel applications, spanning minimally invasive surgery and active drug delivery (Section 3.2 and 3.3).

Nevertheless, synthesis and fabrication of Au NPs (and other nanostructures) of low symmetry, especially with well-defined chirality, remains a tremendous challenge, because energetically favourable geometry at the nanoscale generally exhibits a high degree of symmetry owing to minimisation of surface energy and crystallisation forces.

While it is difficult to synthesise NPs of a chiral shape, classical attempts of investigating chiroptical effect of LSPR relies on the attachment of enantiomerically pure chiral small molecules onto the surface of Au NPs. After functionalisation, the $\mathrm{Au}$ NPs are effectively surrounded by a chiral medium, which will induce chiroptical effects, such as circular dichroism, around the LSPR signal of the Au NP. However, the induced chiroptical effects in the LSPR are typically weak. ${ }^{36-38}$
Other chemical approaches were developed to achieve direct chiroptical effects of LSPR. One effective route is to attach via supramolecular self-assembly Au NPs around chiral molecular templates which are available from natural sources or organic synthesis. Various chiral molecular templates, including amino acids, double-strand DNA and DNA-origami, have been investigated regarding their effectiveness on mediating the formation of chiral assemblies of Au NPs. ${ }^{25,36,39-41}$ Notable success has been attained by using DNA-origami scaffolds owing to their versatility and precision in forming into a wide range of programmable 3-D shapes. Another route towards chiroptical LSPR is to directly grow Au NPs into chiral shapes or with chiral imprints by solution-based synthesis. This can be achieved by growing Au NPs in the presence of chiral-directing agents. Despite being demonstrated in NPs of a few other materials, ${ }^{42-44}$ chemical synthesis of chiral Au NPs has not been reported until very recently. ${ }^{45}$

On the other hand, physical methods, mostly electron-/ionbeam-based lithography, have also been used for the fabrication of diverse 2.5-D and 3-D chiral nanostructures. ${ }^{46}$ These methods can be used to make chiral structures with interesting chiroptical properties, but they often do not permit large (wafer-) scale processing. Yet the possibility of wafer-scale fabrication of chiral $\mathrm{Au}$ NPs has been recently demonstrated using a novel nanofabrication technique (nano-GLAD). ${ }^{31}$ Furthermore, the resultant chiral Au NPs (Fig. 2c) can be easily detached from the wafer surface to form stable nanocolloidal solutions of a practical concentration $\left(10^{10}\right.$ NPs per $\left.\mathrm{mL}\right)$, shedding light on their potential applications as solution-based chiral LSPR biosensors (Section 3.1).

\subsection{Classification of hybrid gold nanoparticles}

Gold nanostructures can be generally classified into three main categories, namely (1) homogeneous Au NPs, (2) heterogeneous $\mathrm{Au}$ NPs and (3) Au NP assemblies. While categories (1) and (2) concern individual NPs, category (3) includes assemblies of Au NPs exhibiting collective properties that are not found in their disassembled counterparts. From (1) to (3), there is a general increase in length scale and material complexity owing to the increase in the number of components made of different materials across the categories. The dimension of symmetry varies rather independently both across and within the categories, with an exception of NPs of very low symmetry, e.g. chiral NPs, which can only be found in the larger length scale regime (Fig. $2 \mathrm{c}, \mathrm{f}$ and $\mathrm{h}$ ). This is due to the relatively low stability of NPs of low symmetry in the small length scale regime owing to surface and lattice effects.

2.4.1 Homogeneous Au NPs. Homogeneous Au NPs refers to NPs that contain only gold within the construct. This is the simplest class of Au NPs, which are chemically stable, highly biocompatible and easy to functionalise using thiol and amine chemistry. The Au NPs are typically stabilised by a selfassembled monolayer of ligand molecules, which prevents them from aggregation and sintering. With the actual wavelength depending on their size and shape, they exhibit well-defined LSPR owing to the absence of other LSPR-damping materials.

At the low end of the length scale, Au clusters and nanoclusters (Fig. 2a) are typically smaller than $5 \mathrm{~nm}$, and exhibit weak or no 
LSPR effects. They are traditionally synthesised from organometallic precursors that require stringent control over reaction conditions. ${ }^{47}$ Recent advances of micro-reactor technology allow them to be made via facile flow synthesis. ${ }^{48,49}$ Au clusters have been shown to have excellent antimicrobial activity. ${ }^{50,51}$

Moving up along the length scale (5-100+ $\mathrm{nm}$ ), there exist a wide variety of $\mathrm{Au}$ nanocrystals with different shapes (Fig. 2b), ${ }^{52}$ ranging from the classical spheres to cubes, plates, rods, wires and stars. Anisotropic shapes with a large aspect ratio can shift the LSPR to NIR wavelength which could be beneficial for in vivo applications.

Moving down the symmetry dimension, there are chiral Au NPs (Fig. 2c) which show unique chiroptical properties around the LSPR wavelength. ${ }^{31,45}$

2.4.2 Heterogeneous Au NPs. Heterogeneous Au NPs are NPs consisting of gold and at least one other material. As a classical example in this category, core-shell NPs (Fig. 2d) are NPs with a core of (typically) gold encapsulated inside a shell of various materials. The shell layer can be made of polymers or inorganic materials and can be used to enhance the chemical and colloidal stability, biocompatibility and anti-fouling properties of the NPs. Furthermore, it can be used to load and release drugs on-demand upon chemical stimuli. ${ }^{53}$

When the core is only partially coated on one side or coated by a different material on each of the two faces, then it will lead to a break of mirror symmetry on the NP, forming Janus NPs (JNPs, Fig. 2e) which are named after the two-faced ancient Roman god. The break of mirror symmetry, when coupled to an appropriate mechanism, can lead to interesting phoretic effects, such as self-electrophoresis in $\mathrm{Au}-\mathrm{Pt} \mathrm{JNPs}^{54}$ and thermophoretic effect in $\mathrm{Au}-\mathrm{SiO}_{2} \mathrm{JNPs}^{55}$ and thermophoresis in $\mathrm{Au}-\mathrm{DNA}$ JNPs. ${ }^{56}$ As a basic asymmetric NP, JNPs can also serve as key intermediates towards more complex asymmetric NPs, such as Au $\mathrm{NP} @ \mathrm{SiO}_{2}$ nanocups. ${ }^{57,58}$

At the higher end of the complexity dimension, heterogeneous $\mathrm{Au}$ NPs can consist of multiple materials (including metals, alloys, ceramics, magnetics and polymers) with different material composition and distribution, which are broadly classified as hybrid Au NPs (Fig. 2f). ${ }^{59-61}$

2.4.3 Au NP assemblies. Au NP assemblies are ensembles of Au NPs exhibiting collective properties and behaviours that are different from the individual building blocks. Additional structural parameters are often required to describe the arrangement of NPs within the assemblies, including interparticle spacing, number of NPs per assembly and degree of ordering.

Metamolecules are discreet assemblies made of a small number $(<50)$ of $\mathrm{Au}$ NPs (Fig. 2g). ${ }^{62}$ This category embraces the simplest forms of NP assemblies (e.g. dimers, trimers, tetramers) which in some cases resemble the shape of small molecules. Typically, they can confine light between their gaps, leading to strong optical field enhancement. ${ }^{63}$ The enhanced optical field can be coupled to vibration of molecules within the gaps and thus making it possible for them to be detected at the single molecular level (Section 3.1.2).

DNA-templated assemblies reside in the higher end of the complexity axis (Fig. 2h). They have a relatively long history, but their development has recently reached a new height, thanks to the DNA origami techniques which can produce highly sophisticated templates for specific attachment of Au NPs. ${ }^{64}$ Their potential applications in the biomedical area are, however, limited by the abundance of deoxyribonuclease (DNase) in the human body that efficiently catalyses the hydrolytic cleavage of phosphodiester linkages in the DNA backbone, thus degrading the DNA scaffolds.

Large scale Au NP assemblies (a.k.a. aggregates) can reach a millimetre in size (Fig. 2i). They are often formed by controlled aggregation of $\mathrm{Au}$ NPs in solution, mediated by gold-ligand bonding, ${ }^{65-68}$ supramolecular complexation ${ }^{59,69}$ electrostatic interactions, ${ }^{70}$ and bio-polymers/objects (incl. proteins, peptides and viruses). ${ }^{64}$

\section{Key features \& applications}

Novel hybrid Au NPs, engineered based on the framework as discussed above, give rise to unique plasmonic features that can serve as a new platform for nanomachines and robots showing unique and multiple functions. They are poised to perform complex tasks ranging from advanced sensing and therapy to other novel applications. The following subsections highlight three key advanced applications of hybrid Au NPs in plasmonic sensing (Section 3.1), plasmonic therapy (Section 3.2), and micro- and nanomachines (Section 3.3). Refer to ref. 4 and 18 for other possible applications using Au NPs, e.g. biomedical imaging.

\subsection{Plasmonic sensing}

In bio-applications, it is desirable to have a local sensor probe for revealing key physiochemical parameters of its immediate surroundings in situ or in vivo, and thus the most prominent application of hybrid Au NPs is plasmonic biosensors. ${ }^{9}$ Since the enhanced optical field is highly localised on the particle, it has only a small penetration depth into the surrounding medium, which makes Au NPs an extremely localised sensor. Crucially, such nano-sensors can be combined with microfluidics or paper-based platform for lateral flow assays (LFAs) ${ }^{71,72}$ or fibre-optics for in situ remote diagnosis. ${ }^{73-76}$ For instance, the LFA with antibody-conjugated Au NPs for pregnancy testing has been successfully integrated in our lives. ${ }^{71}$ Sensing performance in multiplexed point-of-care testing systems is continuously progressing in conjunction with advanced plasmonic sensing schemes using various hybrid Au NPs. Thus, we focus on recent advances in three different generic plasmonic sensing schemes, which have potential use for in vivo or in situ sensing and imaging and to improve the sensing performance of existing assays.

3.1.1 Extinction spectroscopy. LSPR supported by Au NPs is sensitive to the local refractive index $n$ surrounding a nanoparticle (Fig. 3a). ${ }^{6}$ The change in $n$ of the medium, which can also be caused by surface attachment of analyte molecules, leads to the shift in the LSPR peak position, $\lambda^{*}$. The plasmonic sensitivity is given by, ${ }^{7}$

$$
S_{n}=\frac{\mathrm{d} \lambda^{*}}{\mathrm{~d} n}=\frac{\frac{\mathrm{d} \varepsilon_{\mathrm{r}}^{*}}{\mathrm{~d} n}}{\left(\frac{\mathrm{d} \varepsilon_{\mathrm{r}}}{\mathrm{d} \lambda}\right)_{\lambda^{*}}}=\frac{-2 \chi n}{\left(\frac{\mathrm{d} \varepsilon_{\mathrm{r}}}{\mathrm{d} \lambda}\right)_{\lambda^{*}}},
$$


where $\varepsilon_{\mathrm{r}}$ is the real part of the dielectric function of the plasmonic material and $\chi$ is the factor describing the shape of the particle ( 2 for sphere). Conventional LSPR sensors are now poised to detect various cancers (in liver, prostate, ovary, colon, and mutation-induced cancers), AIDS, RNA/DNA viruses, bacteria, inflammation, immune response, microalbuminuria, corneal dystrophy for the purpose of medical diagnosis, drug residues and toxic components in food, and heavy metal ions in the environment. ${ }^{77,78}$ However, so far their sensing performance relies only on pure metal nanoparticles (e.g. Au and Ag) whose sensitivities are typically below $1000 \mathrm{~nm}$ per RIU (Fig. 3b), which are still significantly lower than those of propagating type surface plasmon resonance (SPR) biosensors ( $10000 \mathrm{~nm}$ per RIU). ${ }^{79}$ Hence, for commercialisation, LSPR nanosensors require improved sensitivity and figure of merit (FOM = sensitivity/ FWHM; full width at half maximum, e.g. the bottom panel of Fig. 3a). Attempts in improving sensitivity and FOM have been made via engineering two factors $\chi$ and $\varepsilon_{\mathrm{r}}$, as shown in Fig. $3 \mathrm{~b}$. According to eqn (1) higher $\chi$ leads to enhanced sensitivity, for instance by employing more elongated particles (e.g. rod and prism, from left to right in the framework, e.g. Fig. 2b). ${ }^{80}$ However their sensitivities are typically below $1000 \mathrm{~nm}$ per RIU and the resultant LSPR peaks in the NIR regime are not only hard to resolve via general optical microscopies, but also difficult to incorporate into colorimetric sensing schemes. Meanwhile, decreasing the wavelength dependence of the real part of the material's dielectric constant (proportional to the refractive index of the NP, the denominator in eqn (1)) can also lead to an increase in sensitivity. There are several alloyed plasmonic NPs (e.g. $\mathrm{Au}-\mathrm{Ag},{ }^{81,82} \mathrm{Au}-\mathrm{Cu},{ }^{83}$ and $\left.\mathrm{Au}-\mathrm{Pd}^{84}\right)$, but they show little changes in $\varepsilon_{\mathrm{r}}$, and therefore exhibit limited sensitivity enhancement. Recently, this approach has been achieved by increasing the material complexity (Section 2.2, from top to bottom in the framework in Fig. 2). ${ }^{85}$ For example, mixing a plasmonic metal with a metal possessing low dispersion in $\varepsilon_{\mathrm{r}}$ flattens their effective dielectric dispersion, allowing enhanced sensitivity (>1000 nm per RIU) while keeping their resonance in the visible range. Example combinations are (but not limited to) $\mathrm{Ag}-\mathrm{Ti}^{85}$ and $\mathrm{Ag}-\mathrm{MgF}_{2} \cdot{ }^{86}$ However, the plasmonically inactive materials in the nanoparticle often cause resonance damping, resulting in large FWHM and thus low FOM. ${ }^{87}$ To address the dilemma, exploring the optimal material mixing ratio is necessary.

An elegant way to approach this problem is to introduce shape chirality to the NPs (as discussed in Section 2.3, from left to right in the framework in Fig. 2). This allows the observation of feature-rich plasmonic signals supported by chiral Au NPs using standard chiroptical spectroscopy. ${ }^{31}$ For instance, chiroptical LSPR response can be monitored by circular dichroism (CD) spectroscopy, which measures the differential extinction of the chiral NPs with left- and right-handed circularly polarised light (Fig. 3c, see also Section 3.1.3). ${ }^{88}$ The observed signals possess information about the bipolar nature of CD, from which richer spectral features and zero crossings can be extracted and analysed to yield a narrow effective FWHM and thus high FOM data (lower panel of Fig. 3c). ${ }^{85,89}$ A proof-ofconcept immunoassay based on biotin-avidin binding has been demonstrated using CD spectroscopy on functionalised Ag-Ti nanohelices. ${ }^{85}$ Strictly speaking, here change in the CD spectra is due to the change in $n$ according to the molecular binding on the NPs and unnecessary to involve chirality of molecules (see Section 3.1.3 for chiral molecule sensing). Similar approaches with plasmonic meta-surfaces have been demonstrated in conjunction with magnetic anisotropy, ${ }^{90}$ Fano-type resonance, ${ }^{91}$ or polarisation dependent spectroscopy. ${ }^{92}$ They can increase the sensitivity and FOM, but their mechanisms rely on surface interactions requiring an array
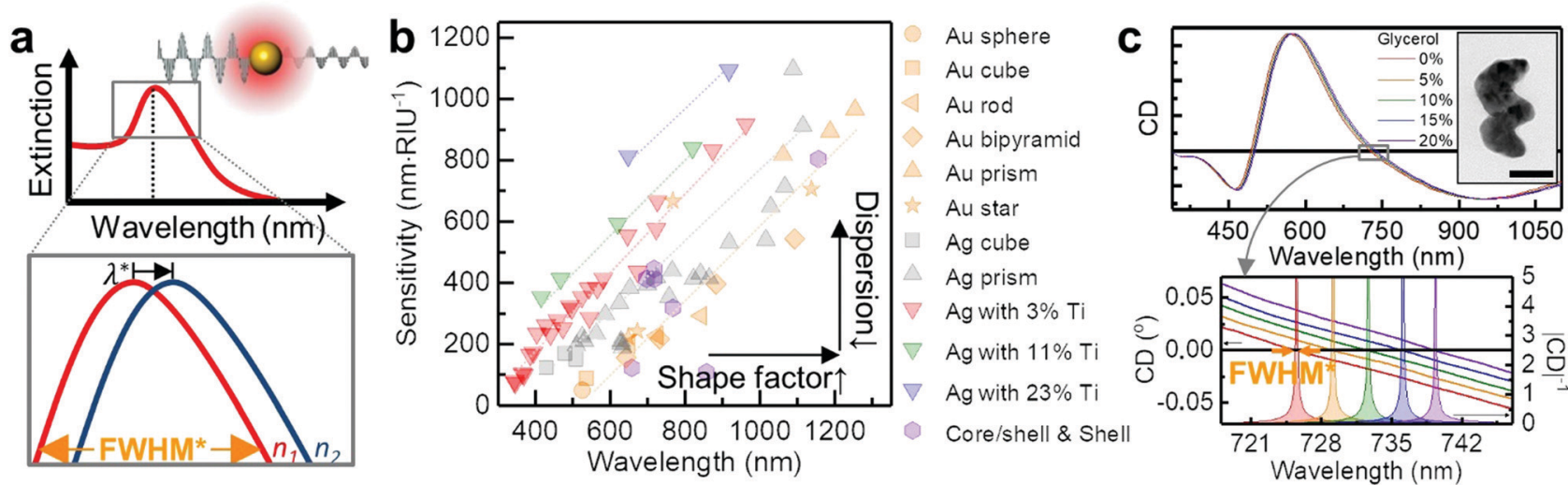

Fig. 3 Plasmonic extinction spectroscopy. (a) Schematic view of plasmonic sensing. Top panel shows the plasmonic resonance of metallic nanoparticles as a function of wavelength. Inset shows a metallic nanosphere interacts with light and generates a detectable absorption peak due to LSPR. The bottom panel shows a zoomed-in region near the peak, illustrating how it shifts in wavelength as the refractive index of the surrounding medium changes $n_{1}<n_{2}$. (b) Sensitivities of plasmonic NPs reported in the literature as a function of the peak wavelength in water. The symbols and colours are different for the shapes and the material composition respectively (Au sphere, ${ }^{80} \mathrm{Au}$ cube, ${ }^{80} \mathrm{Au}$ rod, ${ }^{80} \mathrm{Au}$ bipyramid, ${ }^{80} \mathrm{Au}$ prism, ${ }^{177} \mathrm{Au}$ star, ${ }^{80,178,179} \mathrm{Ag}$ sphere, ${ }^{180,181} \mathrm{Ag}$ cube, ${ }^{182,183} \mathrm{Ag}$ prism, ${ }^{180,184,185}$ core/shell (or shell), ${ }^{186-190}$ and Ag-Ti nanohelices ${ }^{85}$ ). (c) Circular dichroism (CD) spectra of $\mathrm{Ag}$-Ti nanohelices in media of five different refractive indices (red: $0 \%$, orange: $5 \%$, green: $10 \%$, blue: $15 \%$ and violet: $20 \%$ glycerol-water mixtures) over the full spectral range. Inset shows the TEM image of a single $\mathrm{Ag}-\mathrm{Ti}$ nanohelix (scale bar $50 \mathrm{~nm}$ ). The bottom panel shows a detailed plot of the resonance shifts at zero-crossing. The filled curves represent $|C D|^{-1}$. Adapted from ref. 85 with permission. Copyright 2016 Springer Nature. 
of nanostructures, thus limiting modular sensing schemes for in vivo applications.

3.1.2 Vibrational spectroscopy. Plasmonic metamolecules, (Section 2.4.3, Fig. 2g), can induce regions of strong optical near-field enhancement, known as 'hot-spots', Fig. $4 .^{93}$ The field enhancement can be maximised by optimising a ultrathin dielectric gap down to $\sim 1 \mathrm{~nm}$ between plasmonic NPs, which calls for highly precise ordering of individual components (from top to bottom in the framework in Fig. 2). ${ }^{3,63}$ Coupling this nano-gap LSPR to vibronic and electronic transitions with the molecules facilitates sensing from single molecular level $^{94}$ to bulk multiplexing. ${ }^{72,95}$ Two representative schemes are surface enhanced Raman scattering (SERS) ${ }^{96}$ and surface enhanced infrared absorption (SEIRA). ${ }^{97}$ The former utilises the plasmonic field enhancement to amplify the Raman vibrational scattering features of the molecules in the gap (as scale of $10^{4}-10^{8}$ SERS enhancement), enabling molecular 'fingerprinting'. Since SERS enhancement is very sensitive to material purity and structural geometry of the NPs, sophisticated and expensive nanolithography techniques are often required to fabricate high-quality SERS substrates, limiting industrial applications. A recent SERS study, however, focuses on 'lithography-free' self-assembly of plasmonic NPs, in particular with a 'glue' molecule that is not only able to contain single target molecule inside, but also lead to very small nanogap plasmonics. ${ }^{66}$ For example, the nanoparticle-on-mirror (NPoM) construct, two plasmonic systems separated by a host-guest complex (Fig. 4a), gives rise to the strong SERS signal within the volume below $1 \mathrm{~nm}^{3}$ (Fig. 4b), ${ }^{63,98}$ which can now be utilised as a tool for elucidating novel optical dynamics. On the other hand, for industrial applications, extended self-assembly has been developed to achieve the large-scale assembled plasmonic nanoparticles by increasing the material length scale as shown in Fig. 2i (from upper-left to lower-right in the framework in Fig. 2). This offers multiple hot-spots leading to in situ sensing with high sensitivity (left and middle panels of Fig. 4c). ${ }^{66,99}$ Such a system is now able to perform in vivo multiplexing (with animals) and paper-based SERS diagnosis of DNA and protein biomarkers, tumour cells, blood glucose, and bacterial pathogens, ${ }^{100-104}$ potentially via a commercial smartphone (right panel of Fig. 4c). ${ }^{105}$

In contrast, SEIRA signals from ultra-small sample volumes can be achieved by overlapping the plasmonic resonance with the absorption bands of targeted molecules (Fig. 4d). ${ }^{95}$ The resonance mode of the plasmonic antenna can be used to confine the IR right into the nanoscale volume, and can be engineered to overlap with the vibrational signatures of target molecular absorption bands. This allows for simultaneous SEIRA enhancement and detection. Crucially, compared to SERS that necessitates extremely short detection length, SEIRA can still be functional up to several hundred nanometres, making this ideal for the multi-length scale detection ranging from sub-nanometre CO molecules to proteins and lipid membranes over several tens of nanometres. ${ }^{106,107}$ In particular, this technique is expected to be key for understanding the role of protein misfolding in incurable neurodegenerative disorders including Alzheimer's and Parkinson's diseases. ${ }^{95}$ Recently, with an advanced material perspective (from upper-left to lower-right in the framework in Fig. 2h), the barcode-like molecular 2-D imaging based on low loss dielectric SEIRA structures has been developed. ${ }^{108}$

3.1.3 Chiral spectroscopy. Unlike previous sections, here we focus on plasmonic detection of chiral molecules which are abundant in biological systems and medicine - their chirality has been shown to exert major impact on the patient's health. ${ }^{109}$ Left- and right-handed chiral molecules (enantiomers) possess
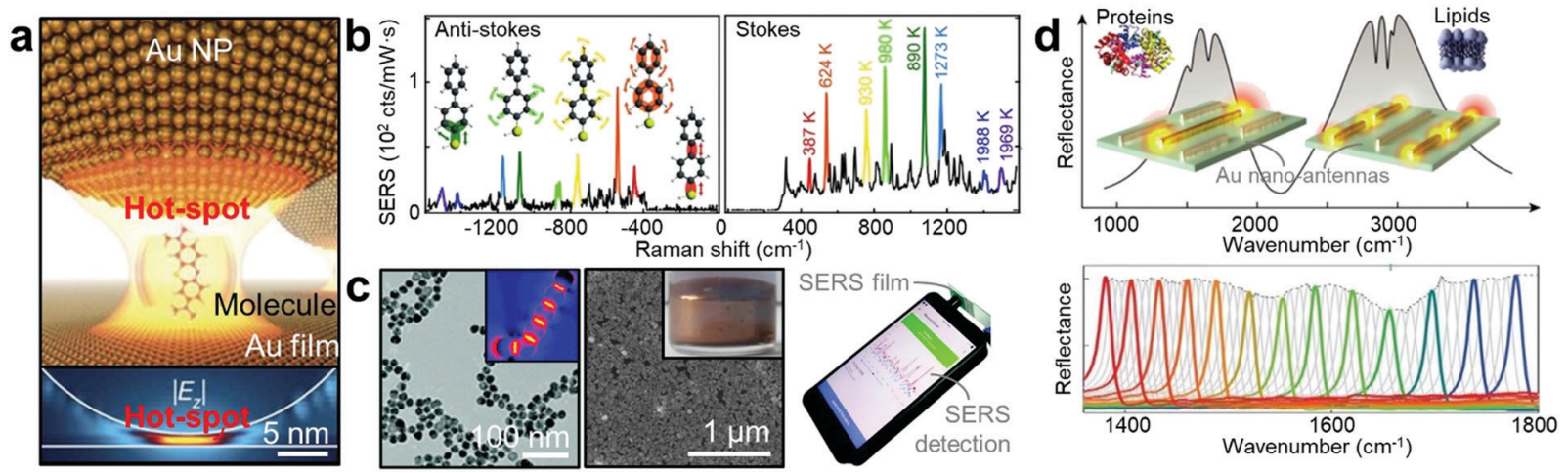

Fig. 4 Plasmonic vibrational spectroscopy. (a) Illustration of a target molecule in the 'glue' molecule in the nanoparticle-on-mirror geometry (top panel). The bottom panel shows the simulated near-field of the coupled gap plasmon. ${ }^{94}$ (b) Anti-Stokes/Stokes spectra at a time when additional picocavityinduced lines are present. Colours correspond to same lines on Stokes and anti-Stokes sides, with vibrational eigenmodes and effective temperatures shown. ${ }^{63}$ (c) Left panel shows the TEM image of Au NPs aggregation with 'glue' molecules. Inset shows the associated numerical simulation of field enhancement. ${ }^{66}$ The middle panel shows the SEM image of the self-assembled gold nanorods (inset: its photo in bulk). ${ }^{99}$ Right panel shows a photo of an on-site SERS measurement using the smartphone-based Raman spectrometer. ${ }^{105}$ (d) Multi-reflection spectra of plasmonic antennas revealing the signal deformation according to lipid membranes and protein molecules in the enhanced optical field (top panel). ${ }^{106}$ Bottom panel shows low loss dielectric meta-pixels that overcome intrinsic metal losses, converting absorption signatures into barcode-like molecular images. ${ }^{108}$ Adapted by permission from the corresponding references. Copyright Spring Nature, American Association for the Advancement of Science, American Chemical Society, Royal Society of Chemistry. 
very similar physical and chemical properties, and the differences in their properties can only be revealed under a chiral influence. For instance, they respond differently to left- and right-circularly polarised light. This is known as optical activity, and is defined as the difference in the complex refractive index of a solution containing one enantiomer of a chiral molecule in excess $\tilde{N}=\tilde{n}_{0} \pm \delta \tilde{n}$ with $\delta \tilde{n}=\delta n+i \delta k$, where $n$ is the refractive index and $k$ is the extinction coefficient of the solution. ${ }^{110}$ The differences in the real $(\delta n)$ and imaginary parts $(\delta k)$, respectively, give rise to circular birefringence (CB) and circular dichroism (CD), and measuring those features provides a direct means of distinguishing the handedness of the chiral molecules. Such differences are of interest in pharmaceutical active compounds because one enantiomer possesses the desired property while another can have unwanted side effects. For example, thalidomide was a treatment (in the late 1950s) for morning sickness, but stopped selling after ca. 10000 children suffering birth defects were born from women taking this drug during pregnancy. ${ }^{111}$ This is due to the interaction of the (S)-(-)-enantiomer with the embryo's prenatal DNA, leading to the birth defects in the form of missing and malformed limbs. ${ }^{46,112}$ Thus measuring the chiroptical effects of chiral compounds has great potential in pharmaceutical and drug industries, but their associated spectral signals are inherently weak since they depend on the change of the optical field of the light across the dimensions of the molecule.

Chiral plasmonic NPs (on the right side of the framework in Fig. 2) promise new sensing schemes that permit the detection of chiral molecules. Since their structural dimensions approach the wavelength of visible light, they give rise to strong chiroptical effects in the visible range of the spectrum. ${ }^{46}$ Impressively, a colloidal solution of chiral NPs show not only several orders of magnitude stronger chiroptical effects than those exhibited by chiral molecules, but also non-linear optical activity, which is not possible to observe from chiral molecules. ${ }^{113}$ We here introduce two representative sensing schemes of chiral molecules using hybrid Au NPs, namely superchiral sensing and chirality transfer. Note that while the former requires chirality of both molecule and NP, only chiral molecule is necessary for the later (i.e. no chiral NP). The former employs the optical field near a chiral plasmonic NP (at the right-corner of the framework in Fig. 2). Such chiral near-field supported by chiral NPs can give rise to an optical chirality surpassing that of circularly polarised light, known as a superchiral field. ${ }^{114}$ This is suggested to distinguish the chiral molecule in the chiral near-field ${ }^{115-117}$ but the underlying mechanism of how the chiral near-field may interact with the chiral molecule and affect the far-field CD spectrum is still under debate. ${ }^{36}$ On the other hand, the approach of chirality transfer utilises Förstertype resonance energy transfer (FRET) with plasmons. ${ }^{118}$ During plasmonic resonance, an achiral NP (i.e. high symmetry at the top-left corner of the framework in Fig. 2) generates a 'hot electron', which is the metastable state of excited electrons, but has no visible optical activity itself. If this plasmonic NP is in contact with a chiral molecule, energy can be transferred to the molecule such that the molecular optical activity can be stimulated. ${ }^{119}$ This could be a flexible and sensitive sensing scheme and recently Raman optical activity (ROA) has been enhanced in this way, ${ }^{120}$ which is analogous to SERS but potentially offers the fingerprinting of molecules in 3-D. They however show yet inherently low signal to noise level, and therefore require extremely active plasmonic NPs to enhance the signal. This challenge might be overcome by using hybrid NPs in the near future.

\subsection{Plasmonic therapy}

Nanoparticle-based platforms have facilitated advances towards noninvasive treatments in medicine, through targeted therapy. ${ }^{121,122}$ In particular, Au NPs can be used for extremely localised thermal therapy, in which light absorption is converted into heat via a process known as plasmonic heating. By integrating multiple functions, hybrid Au NPs can act as multipurpose nanocarriers that perform site-specific and time-controlled drug release and localised thermal therapy. Here we highlight how recent hybrid $\mathrm{Au}$ NPs serve as advanced nano-carriers in therapeutics.

3.2.1 Local therapy using plasmonics. Au NPs can convert absorbed light into thermal energy. Parameters of this process (e.g. wavelength, bandwidth and efficiency) can be tailored through optimising the size, shape and material (imaginary part of the dielectric constant) of the plasmonic NPs. ${ }^{8}$ Under given conditions (including size, number density of Au NPs and light wavelength and power) ${ }^{123}$ the resultant plasmonic heat can elevate the surrounding temperature to over $43{ }^{\circ} \mathrm{C}$, sufficient to damage and kill cancerous tissues by hyperthermia (e.g. MGC803 human gastric cancer cell tumour as shown in Fig. 5a). ${ }^{122,124}$ Crucially, while Au NPs can act as therapeutic agents themselves, they can be further engineered as smart nanocarriers to deliver and release drugs at the desired site and time triggered by plasmonic heating (Fig. 5b). ${ }^{125}$ Here, they generally require material complexity (Section 2.2, from top to bottom in the framework in Fig. 2) in the form of core-shell (Fig. 2d), Janus (Fig. 2e), or assembled NPs (Fig. 2g-i), in conjunction with molecular cargoes such as drugs and genes. For instance, under light exposure, Au NPs decorated with thermoresponsive polymers can generate plasmonic heat, triggering phase transition of the polymers. ${ }^{126}$ Poly( $N$-isopropylacrylamide) (PNIPAM) is a representative example with a phase transition temperature at $\sim 32{ }^{\circ} \mathrm{C}$, leading to large volume change. Genes, nucleic acids, proteins and drugs (e.g. doxorubicin, curcumin, dipyridamole, insulin, 5-fluorouracil) can be trapped/expelled, ${ }^{127}$ thus hybrid systems functionalised with PNIPAM are widely investigated for biomedical applications. ${ }^{128}$ The versatility of hybrid Au NPs extends to multiple and selective release depending on the wave form of the light (Fig. 5c). ${ }^{129}$ Continuous light induces collective heating effects of nanoparticles, causing dehybridisation and release of single-stranded DNA (ssDNA), while pulsed light suppresses such a heating effect and transfers hot electrons to break the Au-S bond, thus releasing double-stranded DNA (dsDNA). Such selective release of DNA (or potentially RNA) can be efficiently harnessed to prohibit transcription or translation of messenger RNA (mRNA), making the NPs useful for applications in gene therapy as well as drug delivery. In the near future, multifunctional hybrid NPs are expected to play a crucial role as versatile 

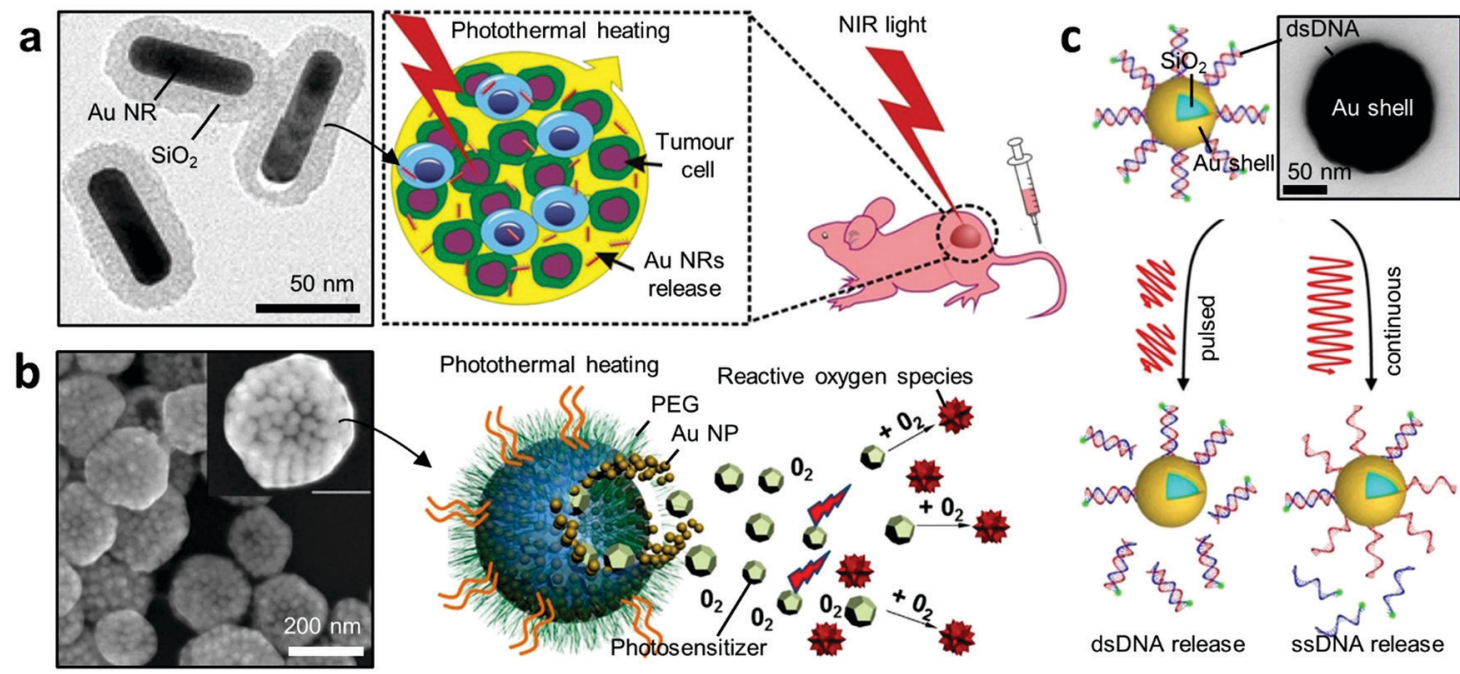

Fig. 5 Plasmonic heating for therapy. (a) Hybrid gold nanomaterials loaded with human cells for target delivery and enhanced photothermal therapy. ${ }^{124}$ Left panel shows the TEM image of the Au core-shell NPs. (b) Photosensitiser-loaded plasmonic gold vesicles for trimodality (fluorescence, thermal, and photoacoustic imaging) guided photo-thermal and dynamic cancer therapy. ${ }^{125}$ (c) NIR-light-induced DNA release. ${ }^{129}$ A continuous light irradiation causes dehybridisation and release of fluorescently tagged single-stranded DNA (ssDNA), while pulsed irradiation results in Au-S bond breakage and release of double-stranded DNA (dsDNA). Adapted by permission from the corresponding references. Copyright American Chemical Society.

nanocarriers for in vivo medical applications, especially for extremely localised non-invasive therapy and surgery.

3.2.2 Targeted drug delivery. Although hybrid Au NPs show promise as nanocarriers for local therapy as discussed above, their use at the clinical level often remains challenging as they suffer from low bio-distribution thus low treatment efficiency. In most cases, distributing nanocarriers at a target site highly relies on their diffusion in the target tissue and/or their surrounding environment. Since cancerous cells and diseased tissues possess complex and heterogeneous physicochemical properties, such a passive diffusional therapy not only becomes less-effective, but may also accompany poor distribution of the NPs in a targeted tissue due to the complexity and heterogeneity of biological tissues. ${ }^{130}$ To address this, hybrid Au NPs are combined with biocompatible matter like stem cells (Fig. 6a, ${ }^{131}$ i.e. increase in the material complexity from top to bottom in the framework in Fig. 2). This combination leads to high bioavailability, good solubility and subsequent distribution into the tissues. ${ }^{132}$ Nevertheless, such diffusion-based processes generally require considerable time, especially in biological materials consisting of multiple membranes that hinder the penetration of substances into the interstitial tumour matrix. ${ }^{133}$ In these cases, the effective dose delivered to the target region is below $1 \% .{ }^{134}$ To confront this issue, controlled target delivery is being investigated. Recently, a simple approach using a plasmonic pen comprising Au NPs linked to a dielectric handle $\left(\mathrm{Al}_{2} \mathrm{O}_{3}\right)$ is used to penetrate the living cell membrane and deliver the genes inside directly (Fig. 6b). ${ }^{135}$ The plasmonically heated Au part allows the whole body of the nanopen to penetrate through the complex membrane and their handle-like body delivers the ssDNA while retaining over $75 \%$ cell viability. Thus, such enhanced targeted delivery systems should facilitate the practical usage of the hybrid $\mathrm{Au}$ NPs in medicine in the near future.

\subsection{Micro/nano-actuators with plasmonics}

As discussed above, the unique optical interaction of hybrid Au NPs with light can be harnessed in numerous ways. Recently this interaction has been adopted for use in various multifunctional nanomachines. ${ }^{136}$ Here we introduce two recent intriguing proof-of-concept applications, namely active nanorheology and active cargo delivery.

3.3.1 Active nanorheology using plasmonics. Anisotropic $\mathrm{Au}$ NPs including rods and helices show polarisation-dependent plasmonic features (from left to right in the framework in Fig. 2). Their polarisability is dominated by their physical long axis, so if the orientation of an anisotropic NP to the incident light is controllable, then the associated plasmonic spectra can be modulated. ${ }^{15}$ The first demonstration relies on strong electric fields to align $\mathrm{Au}$ nanorods. ${ }^{137}$ The long axis of the Au nanorods is aligned along the direction of the external electric field due to a torque exerted by the electric field, and thus their interactions with polarised light can be controlled. Since this scheme requires very high field strength, simpler schemes have been developed in conjunction with magnetic materials that can facilitate alignment by a weak magnetic field. ${ }^{138}$ Combining magnetic features with plasmonics can be achieved by making the multi-layered nanoparticles comprising plasmonic metals with ferromagnetic materials (Fig. 7b and f) ${ }^{23,139-}$ ${ }^{141}$ or by alloying them (Fig. $7 \mathrm{~d},{ }^{142}$ from upper-left to lower-right in the framework in Fig. 2). Due to the natural magnetic anisotropy along the long axis (a.k.a. the easy axis) of the anisotropic NPs, ${ }^{143}$ their long-axis can be aligned to the magnetic moment, $m$ along the magnetic field direction, $B$. 

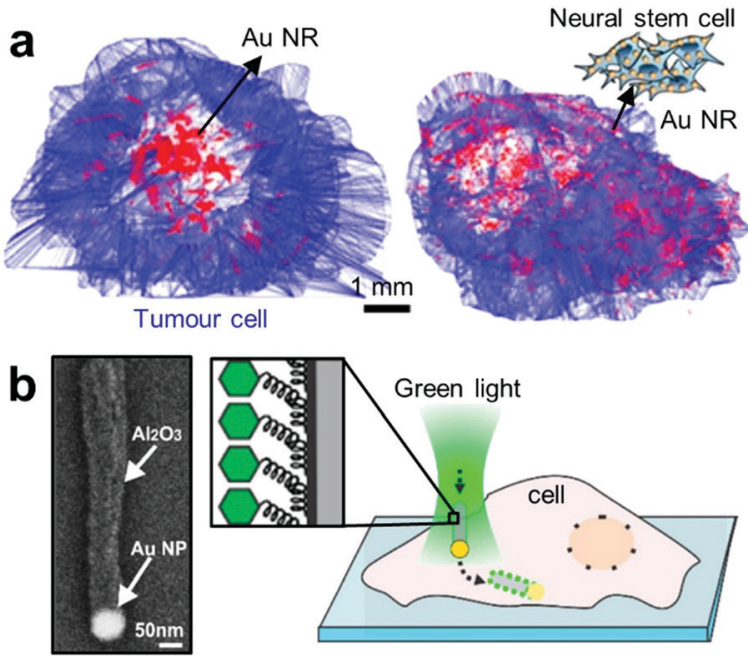

Fig. 6 Advanced target delivery using plasmonics. (a) Distribution of free Au NRs and neural stem cell (NSC)-aided Au NRs after 3 days of intratumoral injection. ${ }^{131}$ (b) Positioning and injection of functionalised plasmonic JNPs into cells. ${ }^{135}$ Left panel shows the SEM micrograph of a JNP consisting of a spherical Au NP ( $\sim 80 \mathrm{~nm}$ ) connected to a dielectric alumina shaft (length: $\sim 500 \mathrm{~nm}$ ). The $\mathrm{Al}_{2} \mathrm{O}_{3}$ part of the JNPs is functionalised with a fluorescently labelled oligo-ssDNA. Adapted by permission from the corresponding references. Copyright American Chemical Society.

The switching property is a crucial basis for their use as nanomechanical probes. Under the rotating magnetic field with frequency $f_{B}$, viscous drag causes a phase lag $\phi$ between $m$ and $B$, which is fixed to its orientation. In the steady state, $\omega=2 \pi f_{B}$, the balance of torques means that the phase angle is related to the drive frequency according to ${ }^{144}$

$$
\sin \phi=\frac{2 \pi S}{m_{\mathrm{r}} B} \eta f_{B},
$$

where $S$ is a constant describing the hydrodynamic shape of the nanoparticle $\left(S=8 \pi R^{3}\right.$ for a sphere) ${ }^{145} m_{\mathrm{r}}$ is the magnetic remanence of a NP, and $\eta$ is the local viscosity. The key insight is that, at the given magnetic field intensity and frequency, the phase lag $\phi$ can be determined by $S$ and $\eta$. This means if one is constant and known, then another can be found by measuring $\phi$. For instance, at the given $\eta$, the total mass change (i.e. $S$ ) of a nanorod induced by molecular interaction (e.g. antibody-antigen reaction) on its surface causes the increase in $\phi$, which directly indicates the amount of antigen on the nanorod, Fig. $7 \mathrm{c}^{140}$

On the other hand, if the probe is protected from any surface interaction (i.e. no change in $S$ ), then viscosity becomes a sole variable affecting $\phi$ and can be measured by tracking $\phi$. This is particularly interesting when the nanoprobes are in the complex fluids containing a mixture of multiple phases. ${ }^{146}$ In fact, since many biological systems fall into this category, this becomes an important consideration for medical applications. Typically, biological complex systems possess a solid phase (e.g. micro-particles and cells or a network of macromolecules) dispersed in a fluid phase, so their macroscopic rheological measurements generally show non-Newtonian viscoelastic behaviour although the liquid phase alone is a simple Newtonian fluid. ${ }^{147}$ It is therefore impossible to measure using conventional macroscopic rheometers the rheological property of the liquid without first isolating the liquid phase from the complex media. However, local measurement of the fluid viscosity can be possible if the total length of the probe is in the nanoscale, which is smaller than the mesh size of the network or the space between the micro particles. ${ }^{148-150}$ In this case, dynamics of the nanoprobes can be decoupled with the dispersed phase, and can be therefore used to measure their surrounding fluidic viscosity, even within complex fluid media. ${ }^{142}$
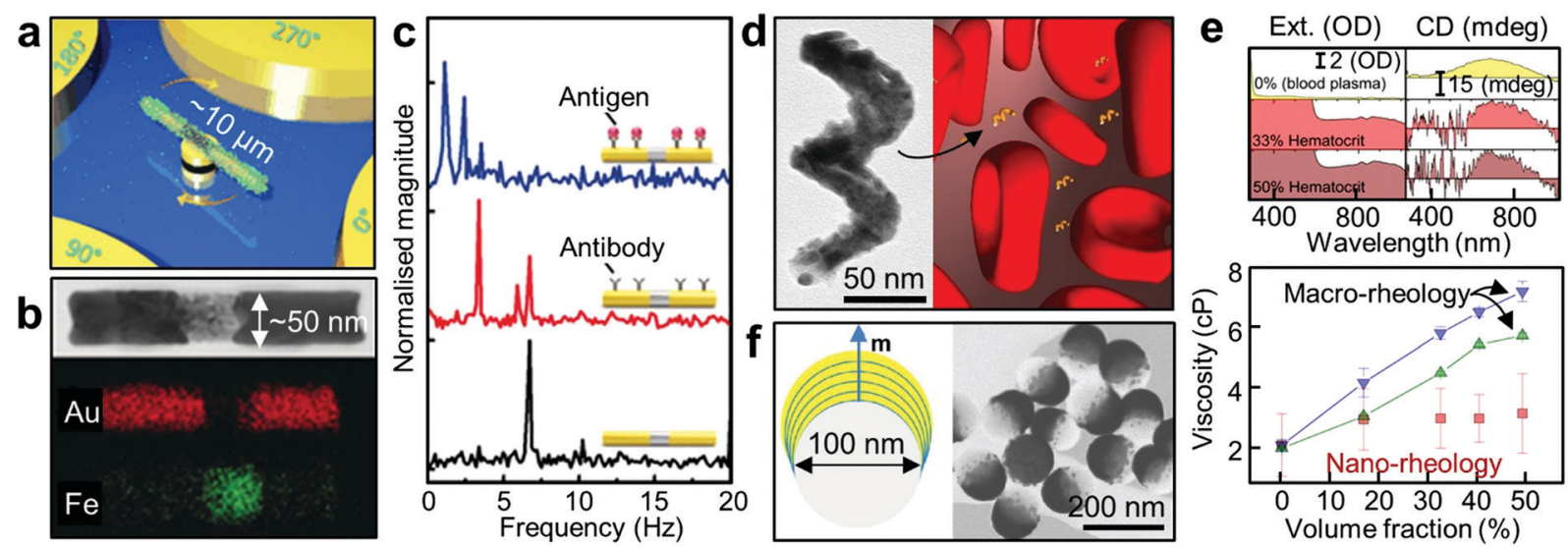

Fig. 7 Active nanorheology using plasmonics. (a) A schematic of a rotating nanomotor sensor. ${ }^{139}$ (b) HR-TEM image of a Au/Fe/Au nanorod and its corresponding elemental false-colour mapping images. (c) FFT plots of the extinction signals of the Au/Fe/Au nanoprobes under a magnetic field rotating at $3.33 \mathrm{~Hz}$ (black: bare probe, red: probe with antibody, blue: probe with antibody-antigen reaction). ${ }^{140}$ (d) TEM image of the 2-turn Au-Fe nanohelix with ca. $170 \mathrm{~nm}$ in height. Schematic depicting a rotating magneto-plasmonic nanoprobe in a complex and optically dense medium (here blood plasma surrounded by erythrocytes). (e) In situ active nanorheology in blood. The top panel shows the extinction and CD spectra of the colloidal Au-Fe nanohelices as a function of wavelength in bovine blood plasma surrounded by the erythrocytes at different volume fractions (top, $0 \%$; middle, $33 \%$; bottom, 50\%). The bottom panel shows the measured dynamic viscosity of the bovine blood plasma in the presence of red blood cells with different hematocrit level. ${ }^{142}$ (f) Schematic of the Co/Au nanodomes with out-of-plane magnetisation (left panel). The right panel shows HR-TEM image of the nanodomes with the Co/Au multilayers. ${ }^{23}$ Adapted by permission from the corresponding references. Copyright Wiley- $\mathrm{VCH}$, American $\mathrm{Chemical}$ Society. 
There exist additional considerations in performing optical measurements in complex media. Optical signals can be hugely attenuated and perturbed by the absorption and scattering of the dispersed solid phase. This explains why it is extremely challenging to extract meaningful optical signals from any optical probes in whole human blood which contains a high level of highly light-absorbing red blood cells. Hence conventional optical sensing is performed in blood plasma without any cells. For direct optical measurement in complex media, innovative approaches are needed to generate optical, spectral or phase contrast against a complex and strongly attenuating background. When coupled with a modulating external magnetic field, polarisation-dependent optical properties of anisotropic NPs can be employed to generate phase contrast in the frequency domain. This approach has been employed in gyromagnetic imaging. Identification and tracking of $\mathrm{Au}$ nanostars with a magnetic core inside tumour cells were achieved using broadband excitation. ${ }^{151}$ Moreover, this approach can be extended by using chiral NPs of Au-Fe (Fig. 7d). In particular, the CD signal of $\mathrm{Au}-\mathrm{Fe}$ nanohelices is background-free as it is insensitive to achiral objects. External magnetic modulation of these magnetic nanohelices allow the direct measurement of blood plasma viscosity in whole blood (optical density of $\sim 3$, Fig. 7e). ${ }^{142}$ This scheme can be further applied to fluids with non-Newtonian properties such as living cells. ${ }^{152}$

3.3.2 Active cargo delivery. The challenges in micro and nanorobots so far have been the development of general methodology for powering these small objects to actively move through the human body and for integrating other functions to perform complicated tasks like their macroscopic counterparts. ${ }^{153}$ Diverse plasmonic-driven actuators have been developed including light-induced rotors using chiral NPs (at the right side of the framework in Fig. 2), ${ }^{154}$ light-driven thermophoretic ${ }^{55,155,156}$ or photocatalytic motors ${ }^{56,157,158}$ based on JNPs (e.g. Au-SiO $\mathrm{Au}$-polymer, and $\mathrm{Au}-\mathrm{TiO}_{2}$ ), and self-propelling actuators powered by repeating the shape deformation (swelling/deswelling) of the thermo-responsive polymer (PNIPAM) surrounding Au NPs in response to the plasmonic heating. ${ }^{159,160}$ These plasmonic-driven actuations, in particular thermophoresis, have been demonstrated in HeLa cells under NIR laser irradiation with no detectable damage to the cells. ${ }^{156}$ Nevertheless, while many micro- and nano-actuators have been reported, ${ }^{161-163}$ only a few schemes have simultaneously demonstrated the capability of self-propelling in biological fluids and performing tasks on top of propulsion. Recently, a hybrid micro-propeller system shows impressive success in addressing these challenges. The system is based on a silica micro-helix scaffold containing a ferromagnetic segment for magnetic propulsion (Fig. 8). ${ }^{35}$ Here, the magnetic moment $m$ along the long axis of the magnetic segment is orthogonal to the long axis of the whole helix body. At the frequency $f_{B}$ of the rotating magnetic field $B$ below the stepout value, the helix rotates synchronously and move forward in liquid. ${ }^{35}$ These micro-propellers are now able to propel in mucus, ${ }^{164}$ living cells, ${ }^{165}$ and eyes (Fig. 8a). ${ }^{166}$ Importantly, the hybrid NPs can also be combined with plasmonic trapping to perform multiple tasks and thus serving as "smart" micro-robots
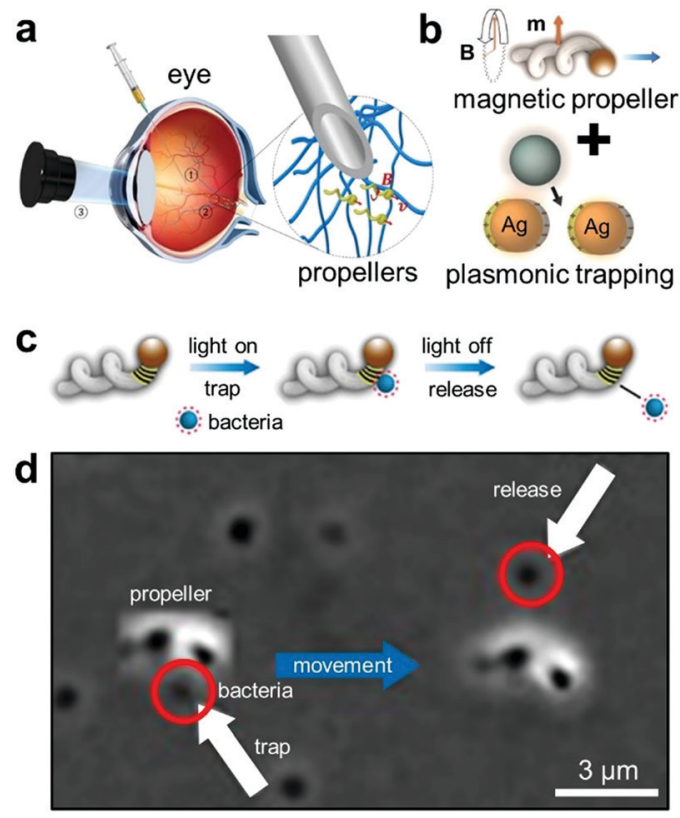

Fig. 8 Micro-actuators with plasmonic trapping. (a) Schematic showing the injection of the helical micro-actuators inside the eye and actuate them via the external magnetic field. ${ }^{166}$ (b) Integration of plasmonic NPs with the helical micro-actuators. ${ }^{167}$ (c) Schematic depicting how the micro-actuator with plasmonic trapping can be functional for active cargo delivery. The bacteria can be trapped and released by light and their actuation can be controlled independently by external magnetic field. (d) Corresponding experimental demonstration. Adapted by permission from the corresponding references. Copyright American Association for the Advancement of Science.

(Fig. 8b). ${ }^{167}$ The plasmonic patches are formed along the body of the helix and can induce hot-spots under light illumination (Fig. 8c). These hot-spots generate optical near-field gradients that attract and trap the target bacteria surrounding the body of the micro-propellers. Since both actuation and trapping are independently controllable by using magnetic and optical fields respectively, the target object (here bacteria) can be delivered to the target area by the aid of plasmonics (Fig. 8d).

\section{Conclusion \& perspective}

Thanks to remarkable recent advances in nanoparticle synthesis and fabrication, underpinned by progress in characterisation techniques and fundamental theories, novel hybrid gold nanoparticles (Au NPs) demonstrate unprecedented physicochemical properties and multi-functionality, promising great potential for diverse biomedical applications.

The development trend of $\mathrm{Au}$ NPs in academia can be reflected in the number of publications containing this and other closely related key words (Fig. 9a). Overall this area of research is booming with number of publications reaching $10^{4}$ per year. It is interesting to note, however, that the share of $\mathrm{Au}$ NPs within the NP literature is slightly declining over the past decade, accompanied by the raise in the share of hybrid Au NPs within the Au NP literature (Fig. 9b). This observation indicates that the focus of the field is shifting towards the development of complex multicomponent NPs. 

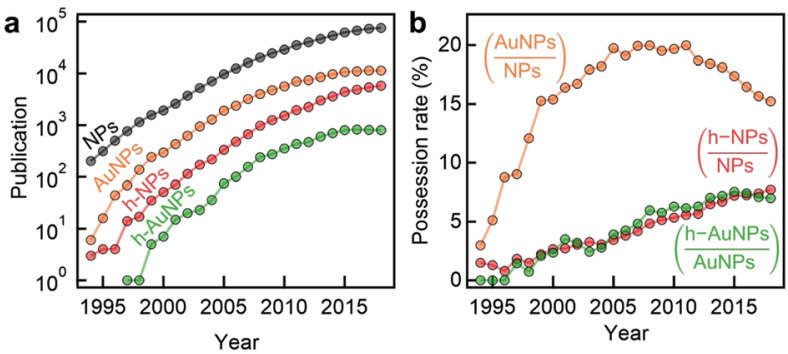

Fig. 9 Growing popularity of hybrid gold nanoparticles. (a) Number of academic articles published containing the terms of nanoparticles (NPs, black), gold nanoparticles (Au NPs, orange), hybrid NPs (red), and hybrid Au NPs (green) since 1994. (b) Possession rate of Au NPs vs. NPs (orange), hybrid NPs vs. NPs (red), and hybrid Au NPs vs. Au NPs (green). Source: Web of Science, as assessed on February 1st 2019.

The rapid and diverse evolution in the hybrid Au NPs motivates us to shape a new classification framework of the hybrid Au NPs based on their fundamental dimensions (length scale, material complexity, and symmetry, Fig. 2). Categorisation systems such as this could help demystify the wide range of materials available and provide a systematic tool for designing new nanomaterials. In the practical aspects, the framework can facilitate convenient comparison and benchmarking of novel Au NPs against well-established Au NP systems. Moreover it could also aid reflection on the future direction of Au NP research with regards to the balance between exploring complex new materials and exploiting well understood existing materials in both fundamental and practical contexts.

In the latter part of the review, we have discussed how the novel physiochemical properties of hybrid Au NPs can potentially be translated to clinical applications, illustrated by recent proof-ofconcept examples in biosensing, photodynamic therapy, gene \& drug delivery, nanorheology and minimally invasive surgery.

Despite the huge volume of research on the topic (as seen in Fig. 9), the number of $\mathrm{Au}$ NP systems finding their way into clinical trials (or other general use) remains insignificant. ${ }^{168}$ Currently, in vivo use of Au NPs have not been approved by the Food and Drug Administration (FDA). ${ }^{169}$ Approval of clinical use of hybrid Au NPs will be even more challenging to obtain, because the combined toxicity from other components is often hard to characterise in the human body. Encapsulating the hybrid $\mathrm{Au}$ NPs within an inert shell (including $\mathrm{PEG}^{170}$ and silica ${ }^{20}$ ) might provide an effective solution, ${ }^{60}$ but their body clearance mechanism is still not fully elucidated, leaving potential risk of bioaccumulation and chronic toxicity. Nevertheless, hybrid Au NPs could find easier translation in in vitro applications. Novel plasmonic sensing schemes (Section 3.1) can be readily implemented in and enhance the capabilities of existing point-of-care technologies, including LFA and hand-held SERS biosensors.

To accelerate the translation and deployment of hybrid $\mathrm{Au}$ NPs and the corresponding technologies in the biomedical context, the field calls for cheap, decentralised and high throughput approaches for toxicity screening and intracellular detection of nanomaterials. ${ }^{171}$ Standard operating procedures (SOPs) of testing should be established within the scientific community and test results should be stored in open-access databases supported by social enterprises or governments initiatives.

On the front of fundamental science, it might be interesting, and perhaps useful, to perform systematic studies on biocompatibility and pharmacokinetics of hybrid Au NPs at the cellular, tissue and animal level, with an aim to extract high-level insights into how hybrid Au NPs interact with biological systems compared to the corresponding individual components. This type of work will benefit from state-of-the-art characterisation techniques. For instance, liquid-cell transmission microscopy (LC TEM) ${ }^{172}$ emerges as a powerful technique for mapping Au NPs within a living cell with unparalleled spatial resolution. Meanwhile optical microscopy based on non-linear optics, e.g. pump-probe four-wave mixing imaging, ${ }^{173}$ offers a promising avenue towards in situ 3-D tracking of single NPs with impressive spatiotemporal resolution.

\section{Conflicts of interest}

There are no conflicts to declare.

\section{Acknowledgements}

TCL is grateful to the Research Project Grant (RPG-2016-393) funded by the Leverhulme Trust. EE and TCL are grateful to the Studentship funded by the A*STAR-UCL Research Attachment Programme through the EPSRC Centre for Doctoral Training in Molecular Modelling and Materials Science (EP/L015862/1). HHJ, EC and TCL are grateful to the Max Planck Society.

\section{Notes and references}

1 Y. Xia, Y. Xiong, B. Lim and S. E. Skrabalak, Angew. Chem., Int. Ed., 2009, 48, 60-103.

2 D. A. Giljohann, D. S. Seferos, W. L. Daniel, M. D. Massich, P. C. Patel and C. A. Mirkin, Angew. Chem., Int. Ed., 2010, 49, 3280-3294.

3 L. Dykman and N. Khlebtsov, Chem. Soc. Rev., 2012, 41, 2256-2282.

4 H. Kim, S. Beack, S. Han, M. Shin, T. Lee, Y. Park, K. S. Kim, A. K. Yetisen, S. H. Yun, W. Kwon and S. K. Hahn, Adv. Mater., 2018, 30, 1701460.

5 X. J. Loh, T.-C. Lee, Q. Dou and G. R. Deen, Biomater. Sci., 2016, 4, 70-86.

6 K. M. Mayer and J. H. Hafner, Chem. Rev., 2011, 111, 3828-3857.

7 M. M. Miller, A. A. Lazarides and N. Carolina, J. Phys. Chem. B, 2005, 109, 21556-21565.

8 A. O. Govorov and H. H. Richardson, Nano Today, 2007, 2, 30-38.

9 J. R. Mejía-Salazar and O. N. Oliveira, Chem. Rev., 2018, 118, 10617-10625.

10 K. Ueno, S. Takabatake, Y. Nishijima, V. Mizeikis, Y. Yokota and H. Misawa, J. Phys. Chem. Lett., 2010, 1, 657-662.

11 L. Lin, M. Wang, X. Peng, E. N. Lissek, Z. Mao, L. Scarabelli, E. Adkins, S. Coskun, H. E. Unalan, B. A. Korgel, L. M. 
Liz-Marzán, E. L. Florin and Y. Zheng, Nat. Photonics, 2018, 12, 195-201.

12 X. Lin, T. Si, Z. Wu and Q. He, Phys. Chem. Chem. Phys., 2017, 19, 23606-23613.

13 S. W. Hsu, A. L. Rodarte, M. Som, G. Arya and A. R. Tao, Chem. Rev., 2018, 118, 3100-3120.

14 Z. Liu, W. R. Leow and X. Chen, Small Methods, 2018, 6, 1800295.

15 N. Jiang, X. Zhuo and J. Wang, Chem. Rev., 2018, 118, 3054-3099.

16 I. Pastoriza-Santos, C. Kinnear, J. Pérez-Juste, P. Mulvaney and L. M. Liz-Marzán, Nat. Rev. Mater., 2018, 3, 375-391.

17 Z. Li and Y. Yin, Adv. Mater., 2019, 1807061, DOI: 10.1002/ adma.201807061.

18 D. Kim, K. Shin, S. G. Kwon and T. Hyeon, Adv. Mater., 2018, 30, 1802309.

19 S. C. Glotzer and M. J. Solomon, Nat. Mater., 2007, 6, 557-562.

20 P. Singh, S. Pandit, V. R. S. S. Mokkapati, A. Garg, V. Ravikumar and I. Mijakovic, Int. J. Mol. Sci., 2018, 19, 1979.

21 Z. Miao, Z. Gao, R. Chen, X. Yu, Z. Su and G. Wei, Curr. Med. Chem., 2018, 25, 1920-1944.

22 F. Hao, C. L. Nehl, J. H. Hafner and P. Nordlander, Nano Lett., 2007, 7, 729-732.

23 Z. Li, A. Lopez-Ortega, A. Aranda-Ramos, J. L. Tajada, J. Sort, C. Nogues, P. Vavassori, J. Nogues and B. Sepulveda, Small, 2018, 14, 1800868.

24 N. Hoshyar, S. Gray, H. Han and G. Bao, Nanomedicine, 2016, 11, 673-692.

25 A. Kuzyk, R. Schreiber, Z. Fan, G. Pardatscher, E. M. Roller, A. Högele, F. C. Simmel, A. O. Govorov and T. Liedl, Nature, 2012, 483, 311-314.

26 E. R. Gray, V. Chudasama, D. A. Richards, J. Kim, B. S. Miller, M. M. Stevens, S. Agarwal, C. N. Loynachan, J. C. Brookes, R. A. McKendry and M. R. Thomas, ACS Nano, 2017, 12, 279-288.

27 W. Lu, N. Jiang and J. Wang, Adv. Mater., 2017, 29, 1604862.

28 J. Peng, H.-H. Jeong, Q. Lin, S. Cormier, H.-L. Liang, M. De Volder, S. Vignolini and J. Baumberg, Sci. Adv., 2019, 5, eaaw2205.

29 S. E. Skrabalak, L. Au, X. Li and Y. Xia, Nat. Protoc., 2007, 2, 2182-2190.

30 R. Klajn, J. F. Stoddart and B. A. Grzybowski, Chem. Soc. Rev., 2010, 39, 2203-2237.

31 A. G. Mark, J. G. Gibbs, T.-C. Lee and P. Fischer, Nat. Mater., 2013, 12, 802-807.

32 J. G. Gibbs, A. G. Mark, T.-C. Lee, S. Eslami, D. Schamel and P. Fischer, Nanoscale, 2014, 6, 9457-9466.

33 P. C. Chen, X. Liu, J. L. Hedrick, Z. Xie, S. Wang, Q. Y. Lin, M. C. Hersam, V. P. Dravid and C. A. Mirkin, Science, 2016, 352, 1565-1569.

34 B. Maruyama, C. A. Mirkin, B. Meckes, K. A. Brown, J. L. Hedrick, J. S. Du, E. J. Kluender, L. M. Moreau and R. Rao, Proc. Natl. Acad. Sci. U. S. A., 2018, 116, 40-45.

35 A. Ghost and P. Fischer, Nano Lett., 2009, 9, 2243-2245.
36 M. Hentschel, M. Schäferling, X. Duan, H. Giessen and N. Liu, Sci. Adv., 2017, 3, e1602735.

37 Y. K. Gun'ko, R. R. Naik, A. O. Govorov, V. A. Gérard, J. M. Slocik and Z. Fan, J. Mater. Chem., 2011, 21, 16806.

38 J. Govan, M. P. Moloney, Y. K. Gun'ko, A. Loudon and M. Mukhina, Nat. Protoc., 2015, 10, 558-573.

39 X. Lan, T. Liu, Z. Wang, A. O. Govorov, H. Yan and Y. Liu, J. Am. Chem. Soc., 2018, 140, 11763-11770.

40 L. V. Besteiro, T. Liedl, A. O. Govorov, R. Schreiber, L. M. Kneer and E.-M. Roller, ACS Nano, 2018, 12, 9110-9115.

41 L. Lermusiaux and S. Bidault, Langmuir, 2018, 34, 14946-14953.

42 Y. Wu, S. W. Sides, J. Tang, J. Wang, G. Cheng, G. H. Fredrickson, K. Katsov, G. D. Stucky and M. Moskovits, Nat. Mater., 2004, 3, 816-822.

43 Y. Yan, Y. Qiao, Z. Yang, J. Liu, Y. Lin, J. Huang, Y. Wang and J. Zhou, Nano Lett., 2009, 9, 4500-4504.

44 C. Li, K. Deng, Z. Tang and L. Jiang, J. Am. Chem. Soc., 2010, 132, 8202-8209.

45 H. E. Lee, H. Y. Ahn, J. Mun, Y. Y. Lee, M. Kim, N. H. Cho, K. Chang, W. S. Kim, J. Rho and K. T. Nam, Nature, 2018, 556, 360-364.

46 V. K. Valev, J. J. Baumberg, C. Sibilia and T. Verbiest, $A d v$. Mater., 2013, 25, 2517-2534.

47 T. Udayabhaskararao, P. K. Kundu, J. Ahrens and R. Klajn, Chem. Phys. Chem., 2016, 17, 1805-1809.

48 R. Baber, L. Mazzei, N. T. K. Thanh and A. Gavriilidis, Nanoscale, 2017, 9, 14149-14161.

49 H. Huang, H. du Toit, M. O. Besenhard, S. Ben-Jaber, P. Dobson, I. Parkin and A. Gavriilidis, Chem. Eng. Sci., 2018, 189, 422-430.

50 S. Noimark, E. Allan and I. P. Parkin, Chem. Sci., 2014, 5, 2216-2223.

51 Z. Jiang, A. Sahar, X. Li, S. M. Robinson, V. M. Rotello, K. Saha, M. A. Riley, D. F. Moyano and A. Gupta, ACS Nano, 2014, 8, 10682-10686.

52 B. P. Khanal and E. R. Zubarev, ACS Nano, 2019, 13, 2370-2378.

53 Q. Lin, A. Poma, X. J. Loh, G. Battaglia, T.-C. Lee, E. Ellis, K. Zhang and E. Ye, J. Mater. Chem. B, 2017, 5, 4421-4425.

54 T.-C. Lee, M. Alarcón-Correa, C. Miksch, K. Hahn, J. G. Gibbs and P. Fischer, Nano Lett., 2014, 14, 2407-2412.

55 M. Xuan, Z. Wu, J. Shao, L. Dai, T. Si and Q. He, J. Am. Chem. Soc., 2016, 138, 6492-6497.

56 X. Hu, C. Fan, K. Wang, J. Ren, D. Li, Y. Gao, W. Qin, J. Shi, F. Wang and T. Peng, Angew. Chem., Int. Ed., 2016, 56, 515-518.

57 M. Alarcõn-Correa, T.-C. Lee and P. Fischer, Angew. Chem., Int. Ed., 2015, 54, 6730-6734.

58 H.-H. Jeong, A. G. Mark, T.-C. Lee, K. Son, W. Chen, M. Alarcón-Correa, I. Kim, G. Schütz and P. Fischer, Adv. Sci., 2015, 2, 1500016.

59 A. D. Celiz, T.-C. Lee and O. A. Scherman, Adv. Mater., 2009, 21, 3937-3940.

60 H.-H. Jeong, M. Alarcón-Correa, A. G. Mark, K. Son, T.-C. Lee and P. Fischer, Adv. Sci., 2017, 4, 1700234. 
61 S. T. Jones, O. A. Scherman, A. Kuhn, R. J. Coulston, S. Mahajan, V. Lapeyre, P. Garrigue, O. Nicoletti, G. Loget, R. W. Taylor, T.-C. Lee, J. J. Baumberg and P. A. Midgley, Small, 2012, 8, 2698-2703.

62 G. Haran and L. Chuntonov, Chem. Rev., 2018, 118, 5539-5580.

63 F. Benz, M. K. Schmidt, A. Dreismann, R. Chikkaraddy, Y. Zhang, A. Demetriadou, C. Carnegie, H. Ohadi, B. De Nijs, R. Esteban, J. Aizpurua and J. J. Baumberg, Science, 2016, 354, 726-729.

64 Y. Ofir, B. Samanta and V. M. Rotello, Chem. Soc. Rev., 2008, 37, 1814-1825.

65 T.-C. Lee and O. A. Scherman, Chem. Commun., 2010, 46, 2438-2440.

66 R. W. Taylor, T.-C. Lee, O. A. Scherman, R. Esteban, J. Aizpurua, F. M. Huang, J. J. Baumberg and S. Mahajan, ACS Nano, 2011, 5, 3878-3887.

67 T.-C. Lee and O. A. Scherman, Chem. - Eur. J., 2012, 18, 1628-1633.

68 S. Mahajan, T.-C. Lee, F. Biedermann, J. T. Hugall, J. J. Baumberg and O. A. Scherman, Phys. Chem. Chem. Phys., 2010, 12, 10429-10433.

69 R. J. Coulston, S. T. Jones, T.-C. Lee, E. A. Appel and O. A. Scherman, Chem. Commun., 2011, 47, 164-166.

70 T. Udayabhaskararao, R. Popovitz-Biro, M. Coronado-Puchau, P. Král, L. Houben, R. Klajn, J. Langer, L. Vuković, S. Bals, T. Altantzis and L. M. Liz-Marzán, Science, 2017, 358, 514-518.

71 K. M. Koczula and A. Gallotta, Essays Biochem., 2016, 60, 111-120.

72 H. Tang, C. Zhu, G. Meng and N. Wu, J. Electrochem. Soc., 2018, 165, B3098-B3118.

73 H.-H. Jeong, N. Erdene, J.-H. Park, D.-H. Jeong, H.-Y. Lee and S.-K. Lee, Biosens. Bioelectron., 2013, 39, 346-351.

74 H.-H. Jeong, N. Erdene, D.-H. Jeong, S.-K. Lee and J.-H. Park, Opt. Eng., 2011, 50, 124405.

75 H.-M. Kim, J.-H. Park, D. H. Jeong, H.-Y. Lee and S.-K. Lee, Sens. Actuators, B, 2018, 273, 891-898.

76 H.-M. Kim, M. Uh, D. H. Jeong, J.-H. Park and S.-K. Lee, Sens. Actuators, B, 2018, 280, 183-191.

77 C. D. Geddes, Reviews in Plasmonics 2016, Springer, 2016, vol. 2015.

78 B. Špačková, P. Wrobel, M. Bocková and J. Homola, Proc. IEEE, 2016, 104, 2380-2408.

79 J. Homola and M. Piliarik, Surface Plasmon Resonance Based Sensors, Springer, Berlin, Heidelberg, 2006, pp. 45-67.

80 H. Chen, X. Kou, Z. Yang, W. Ni and J. Wang, Langmuir, 2008, 24, 5233-5237.

81 M. S. Shore, J. Wang, A. C. Johnston-Peck, A. L. Oldenburg and J. B. Tracy, Small, 2011, 7, 230-234.

82 M. Verma, M. B. Newmai and P. Senthil Kumar, Dalton Trans., 2017, 46, 9664-9677.

83 G. Guisbiers, S. Mejia-Rosales, S. Khanal, F. Ruiz-Zepeda, R. L. Whetten and M. José-Yacaman, Nano Lett., 2014, 14, 6718-6726.

84 M. Matuschek, D. P. Singh, H.-H. Jeong, M. Nesterov, T. Weiss, P. Fischer, F. Neubrech and N. Liu, Small, 2018, 14, 1702990.
85 H.-H. Jeong, A. G. Mark, M. Alarcón-Correa, I. Kim, P. Oswald, T.-C. Lee and P. Fischer, Nat. Commun., 2016, 7, 11331.

86 S. Larson and Y. Zhao, J. Phys. Chem. C, 2018, 122, 7374-7381.

87 T. G. Habteyes, S. Dhuey, E. Wood, D. Gargas, S. Cabrini, P. J. Schuck, A. P. Alivisatos and S. R. Leone, ACS Nano, 2012, 6, 5702-5709.

88 J. G. Gibbs, A. G. Mark, S. Eslami and P. Fischer, Appl. Phys. Lett., 2013, 103, 213101.

89 H.-H. Jeong, A. G. Mark and P. Fischer, Chem. Commun., 2016, 52, 12179-12182.

90 N. Maccaferri, K. E. Gregorczyk, T. V. A. G. De Oliveira, M. Kataja, S. Van Dijken, Z. Pirzadeh, A. Dmitriev, J. Åkerman, M. Knez and P. Vavassori, Nat. Commun., 2015, 6, 6150.

91 Y. Shen, J. Zhou, T. Liu, Y. Tao, R. Jiang, M. Liu, G. Xiao, J. Zhu, Z. K. Zhou, X. Wang, C. Jin and J. Wang, Nat. Commun., 2013, 4, 2381.

92 B. Ai, P. Basnet, S. Larson, W. Ingram and Y. Zhao, Nanoscale, 2017, 9, 14710-14721.

93 W. Zhu, R. Esteban, A. G. Borisov, J. J. Baumberg, P. Nordlander, H. J. Lezec, J. Aizpurua and K. B. Crozier, Nat. Commun., 2016, 7, 11495.

94 R. Chikkaraddy, B. De Nijs, F. Benz, S. J. Barrow, O. A. Scherman, E. Rosta, A. Demetriadou, P. Fox, O. Hess and J. J. Baumberg, Nature, 2016, 535, 127-130.

95 S.-H. Oh and H. Altug, Nat. Commun., 2018, 9, 5263.

96 S. Schlücker, Angew. Chem., Int. Ed., 2014, 53, 4756-4795.

97 F. Neubrech, A. Pucci, T. W. Cornelius, S. Karim, A. GarcíaEtxarri and J. Aizpurua, Phys. Rev. Lett., 2008, 101, 157403.

98 C. Carnegie, J. P. Griffiths, B. de Nijs, C. Readman, R. Chikkaraddy, W. D. Deacon, Y. Zhang, I. Szabo, E. Rosta, J. Aizpurua and J. J. Baumberg, J. Phys. Chem. Lett., 2018, 9, 7146-7151.

99 L. Tian, M. Su, F. Yu, Y. Xu, X. Li, L. Li, H. Liu and W. Tan, Nat. Commun., 2018, 9, 3642.

100 C. Zong, M. Xu, L. J. Xu, T. Wei, X. Ma, X. S. Zheng, R. Hu and B. Ren, Chem. Rev., 2018, 118, 4946-4980.

101 L. A. Lane, X. Qian and S. Nie, Chem. Rev., 2015, 115, 10489-10529.

102 R. Wang, K. Kim, N. Choi, X. Wang, J. Lee, J. H. Jeon, G. Rhie and J. Choo, Sens. Actuators, B, 2018, 270, 72-79.

103 M. Lee, K. Oh, H. K. Choi, S. G. Lee, H. J. Youn, H. L. Lee and D. H. Jeong, ACS Sens., 2018, 3, 151-159.

104 X. Gao, P. Zheng, S. Kasani, S. Wu, F. Yang, S. Lewis, S. Nayeem, E. B. Engler-Chiurazzi, J. G. Wigginton, J. W. Simpkins and N. Wu, Anal. Chem., 2017, 89, 10104-10110.

105 F. Zeng, T. Mou, C. Zhang, X. Huang, B. Wang, X. Ma and J. Guo, Analyst, 2018, 144, 137-142.

106 D. Rodrigo, A. Tittl, N. Ait-Bouziad, A. John-Herpin, O. Limaj, C. Kelly, D. Yoo, N. J. Wittenberg, S. H. Oh, H. A. Lashuel and H. Altug, Nat. Commun., 2018, 9, 2160.

107 F. Neubrech, C. Huck, K. Weber, A. Pucci and H. Giessen, Chem. Rev., 2017, 117, 5110-5145.

108 A. Tittl, A. Leitis, M. Liu, F. Yesilkoy, D. Y. Choi, D. N. Neshev, Y. S. Kivshar and H. Altug, Science, 2018, 360, 1105-1109. 
109 A. J. Hutt and S. C. Tan, Drugs, 1996, 52, 1-12.

110 Circular Dichroism and the Conformational Analysis of Biomolecules, ed. G. D. Fasman, Springer US, Boston, MA, 1996.

111 M. Melchert and A. List, Int. J. Biochem. Cell Biol., 2007, 39, 1489-1499.

112 K. Hotta, Y. Yamaguchi, Y. Imamura, H. Handa, T. Ogura, T. Ito, H. Ando and T. Suzuki, Science, 2010, 327, 1345-1350.

113 J. T. T. Collins, K. R. R. Rusimova, D. C. C. Hooper, H.-H. Jeong, L. Ohnoutek, F. Pradaux-Caggiano, T. Verbiest, D. R. R. Carbery, P. Fischer and V. K. K. Valev, Phys. Rev. $X, 2019$, 9, 011024.

114 Y. Tang and A. E. Cohen, Science, 2011, 332, 333-336.

115 E. Hendry, T. Carpy, J. Johnston, M. Popland, R. V. Mikhaylovskiy, A. J. Lapthorn, S. M. Kelly, L. D. Barron, N. Gadegaard and M. Kadodwala, Nat. Nanotechnol., 2010, 5, 783-787.

116 R. Tullius, G. W. Platt, L. Khosravi Khorashad, N. Gadegaard, A. J. Lapthorn, V. M. Rotello, G. Cooke, L. D. Barron, A. O. Govorov, A. S. Karimullah and M. Kadodwala, ACS Nano, 2017, 11, 12049-12056.

117 Y. Zhao, A. N. Askarpour, L. Sun, J. Shi, X. Li and A. Alù, Nat. Commun., 2017, 8, 14180.

118 J. Li, S. K. Cushing, F. Meng, T. R. Senty, A. D. Bristow and N. Wu, Nat. Photonics, 2015, 9, 601-607.

119 A. Ben-Moshe, B. M. Maoz, A. O. Govorov and G. Markovich, Chem. Soc. Rev., 2013, 42, 7028-7041.

120 S. Ostovar Pour, L. Rocks, K. Faulds, D. Graham, V. Parchaňský, P. Bouř and E. W. Blanch, Nat. Chem., 2015, 7, 591-596.

121 H. Xin, B. Namgung and L. P. Lee, Nat. Rev. Mater., 2018, 3, 228-243.

122 S. H. Yun and S. J. J. Kwok, Nat. Biomed. Eng., 2017, 1, 0008.

123 H. S. Kim and D. Y. Lee, J. Pharm. Invest., 2017, 47, 19-26.

124 Y. Liu, M. Yang, J. Zhang, X. Zhi, C. Li, C. Zhang, F. Pan, K. Wang, Y. Yang, J. Martinez De La Fuentea and D. Cui, ACS Nano, 2016, 10, 2375-2385.

125 J. Lin, S. Wang, P. Huang, Z. Wang, S. Chen, G. Niu, W. Li, J. He, D. Cui, G. Lu, X. Chen and Z. Nie, ACS Nano, 2013, 7, 5320-5329.

126 S. Cormier, T. Ding, V. Turek and J. J. Baumberg, Adv. Opt. Mater., 2018, 6, 1701281.

127 M. Molina, M. Asadian-Birjand, J. Balach, J. Bergueiro, E. Miceli and M. Calderón, Chem. Soc. Rev., 2015, 44, 6161-6186.

128 M. Karimi, A. Ghasemi, P. Sahandi Zangabad, R. Rahighi, S. M. Moosavi Basri, H. Mirshekari, M. Amiri, Z. Shafaei Pishabad, A. Aslani, M. Bozorgomid, D. Ghosh, A. Beyzavi, A. Vaseghi, A. R. Aref, L. Haghani, S. Bahrami and M. R. Hamblin, Chem. Soc. Rev., 2016, 45, 1457-1501.

129 A. M. Goodman, N. J. Hogan, S. Gottheim, C. Li, S. E. Clare and N. J. Halas, ACS Nano, 2017, 11, 171-179.

130 J. Shi, P. W. Kantoff, R. Wooster and O. C. Farokhzad, Nat. Rev. Cancer, 2017, 17, 20-37.

131 R. Mooney, L. Roma, D. Zhao, D. Van Haute, E. Garcia, S. U. Kim, A. J. Annala, K. S. Aboody and J. M. Berlin, ACS Nano, 2014, 8, 12450-12460.
132 H. Maeda, Adv. Drug Delivery Rev., 2015, 91, 3-6.

133 D. Rosenblum, N. Joshi, W. Tao, J. M. Karp and D. Peer, Nat. Commun., 2018, 9, 1410.

134 H. F. Dvorak, A. J. Tavares, S. Ohta, J. Audet, W. C. W. Chan, S. Wilhelm and Q. Dai, Nat. Rev. Mater., 2016, 1, 16014.

135 C. M. Maier, M. A. Huergo, S. Milosevic, C. Pernpeintner, M. Li, D. P. Singh, D. Walker, P. Fischer, J. Feldmann and T. Lohmueller, Nano Lett., 2018, 18, 7935-7941.

136 E. Ellis, S. Moorthy, W. I. K. Chio and T.-C. Lee, Chem. Commun., 2018, 54, 4075-4090.

137 B. M. I. van der Zande, G. J. M. Koper and H. N. W. Lekkerkerker, J. Phys. Chem. B, 1999, 103, 5754-5760.

138 M. Wang, C. Gao, L. He, Q. Lu, J. Zhang, C. Tang, S. Zorba and Y. Yin, J. Am. Chem. Soc., 2013, 135, 15302-15305.

139 X. Xu, K. Kim and D. Fan, Angew. Chem., Int. Ed., 2015, 54, 2525-2529.

140 I. Jung, H. Yoo, H. J. Jang, S. Cho, K. Lee, S. Hong and S. Park, Angew. Chem., Int. Ed., 2018, 57, 1841-1845.

141 D. Seo, K. M. Southard, J. W. Kim, H. J. Lee, J. Farlow, J. U. Lee, D. B. Litt, T. Haas, A. P. Alivisatos, J. Cheon, Z. J. Gartner and Y. W. Jun, Cell, 2016, 165, 1507-1518.

142 H.-H. Jeong, A. G. Mark, T.-C. Lee, M. A. Alarcón-Correa, S. Eslami, T. Qiu, J. G. Gibbs and P. Fischer, Nano Lett., 2016, 16, 4887-4894.

143 C. Phatak, Y. Liu, E. B. Gulsoy, D. Schmidt, E. FrankeSchubert and A. Petford-Long, Nano Lett., 2014, 14, 759-764.

144 L. Chevry, N. K. Sampathkumar, A. Cebers and J. F. Berret, Phys. Rev. E: Stat., Nonlinear, Soft Matter Phys., 2013, 88, 62306.

145 E. Lauga and T. R. Powers, Rep. Prog. Phys., 2009, 72, 096601.

146 R. Splinter, Handbook of Physics in Medicine and Biology, Springer New York, New York, NY, 2010.

147 I. M. Krieger and T. J. Dougherty, Trans. Soc. Rheol., 1959, 3, 137-152.

148 L. M. Ensign, B. C. Tang, Y. Y. Wang, T. A. Tse, T. Hoen, R. Cone and J. Hanes, Sci. Transl. Med., 2012, 4, 138 ra79.

149 Q. Xu, N. J. Boylan, J. S. Suk, Y. Y. Wang, E. A. Nance, J. C. Yang, P. J. McDonnell, R. A. Cone, E. J. Duh and J. Hanes, J. Controlled Release, 2013, 167, 76-84.

150 D. Schamel, A. G. Mark, J. G. Gibbs, C. Miksch, K. I. Morozov, A. M. Leshansky and P. Fischer, ACS Nano, 2014, 8, 8794-8801.

151 Q. Wei, H. M. Song, A. P. Leonov, J. A. Hale, D. Oh, Q. K. Ong, K. Ritchie and A. Wei, J. Am. Chem. Soc., 2009, 131, 9728-9734.

152 J. F. Berret, Nat. Commun., 2016, 7, 10134.

153 S. Palagi and P. Fischer, Nat. Rev. Mater., 2018, 3, 113-124.

154 M. Liu, T. Zentgraf, Y. Liu, G. Bartal and X. Zhang, Nat. Nanotechnol., 2010, 5, 570-573.

155 I. Kaminer, M. Soljačić, H. Buljan, O. Ilic and Y. Lahini, ACS Photonics, 2016, 3, 197-202.

156 Y. Wu, T. Si, J. Shao, Z. Wu and Q. He, Nano Res., 2016, 9, 3747-3756. 
157 B. Jang, A. Hong, H. E. Kang, C. Alcantara, S. Charreyron, F. Mushtaq, E. Pellicer, R. Büchel, J. Sort, S. S. Lee, B. J. Nelson and S. Pané, ACS Nano, 2017, 11, 6146-6154.

158 Q. Zhang, R. Dong, Y. Wu, W. Gao, Z. He and B. Ren, ACS Appl. Mater. Interfaces, 2017, 9, 4674-4683.

159 A. Mourran, H. Zhang, R. Vinokur and M. Möller, Adv. Mater., 2017, 29, 1604825.

160 V. K. Valev, J. J. Baumberg, O. A. Scherman, T. Ding, C. J. Forman, D. Frenkel, S. K. Smoukov and A. R. Salmon, Proc. Natl. Acad. Sci. U. S. A., 2016, 113, 5503-5507.

161 M. Luo, Y. Feng, T. Wang and J. Guan, Adv. Funct. Mater., 2018, 28, 1706100.

162 H. Wang and M. Pumera, Adv. Funct. Mater., 2018, 28, 1705421.

163 K. Kim, J. Guo, Z. Liang and D. Fan, Adv. Funct. Mater., 2018, 28, 1705867.

164 D. Walker, B. T. Käsdorf, H.-H. Jeong, O. Lieleg and P. Fischer, Sci. Adv., 2015, 1, e1500501.

165 M. Pal, N. Somalwar, A. Singh, R. Bhat, S. M. Eswarappa, D. K. Saini and A. Ghosh, Adv. Mater., 2018, 30, 1800429.

166 Z. Wu, J. Troll, H.-H. Jeong, Q. Wei, M. Stang, F. Ziemssen, Z. Wang, M. Dong, S. Schnichels and T. Qiu, Sci. Adv., 2018, 4, eaat4388.

167 S. Ghosh and A. Ghosh, Sci. Rob., 2018, 3, eaaq0076.

168 A. C. Anselmo and S. Mitragotri, Bioeng. Transl. Med., 2016, 1, 10-29.

169 D. Bobo, K. J. Robinson, J. Islam, K. J. Thurecht and S. R. Corrie, Pharm. Res., 2016, 33, 2373-2387.

170 S. K. Libutti, G. F. Paciotti, A. A. Byrnes, H. R. Alexander, W. E. Gannon, M. Walker, G. D. Seidel, N. Yuldasheva and L. Tamarkin, Clin. Cancer Res., 2010, 16, 6139-6149.

171 A. R. Collins, B. Annangi, L. Rubio, R. Marcos, M. Dorn, C. Merker, I. Estrela-Lopis, M. R. Cimpan, M. Ibrahim, E. Cimpan, M. Ostermann, A. Sauter, N. El Yamani, S. Shaposhnikov, S. Chevillard, V. Paget, R. Grall, J. Delic, F. G. De-Cerio, B. Suarez-Merino, V. Fessard, K. N. Hogeveen, L. M. Fjellsbø, E. R. Pran, T. Brzicova, J. Topinka, M. J. Silva, P. E. Leite, A. R. Ribeiro, J. M. Granjeiro, R. Grafström, A. Prina-Mello and M. Dusinska, Wiley Interdiscip. Rev.: Nanomed. Nanobiotechnol., 2017, 9, e1413.
172 N. De Jonge and F. M. Ross, Nat. Nanotechnol., 2011, 6, 695-704.

173 V. Kravtsov, R. Ulbricht, J. M. Atkin and M. B. Raschke, Nat. Nanotechnol., 2016, 11, 459-464.

174 Q. Zhang, K. Kusada, D. Wu, T. Yamamoto, T. Toriyama, S. Matsumura, S. Kawaguchi, Y. Kubota and H. Kitagawa, Nat. Commun., 2018, 9, 510.

175 N. J. Greybush, I. Liberal, L. Malassis, J. M. Kikkawa, N. Engheta, C. B. Murray and C. R. Kagan, ACS Nano, 2017, 11, 2917-2927.

176 X. Qiao, Z. Xue, L. Liu, K. Liu and T. Wang, Adv. Mater., 2018, 31, 1804275.

177 R. Liu, J. H. Zhou, Z. K. Zhou, X. Jiang, J. Liu, G. Liu and X. H. Wang, Nanoscale, 2014, 6, 13145-13153.

178 C. L. Nehl, H. Liao and J. H. Hafner, Nano Lett., 2006, 6, 683-688.

179 S. K. Dondapati, T. K. Sau, C. Hrelescu, T. A. Klar, F. D. Stefani and J. Feldmann, ACS Nano, 2010, 4, 6318-6322.

180 J. J. Mock, D. R. Smith and S. Schultz, Nano Lett., 2003, 3, 485-491.

181 A. D. McFarland and R. P. Van Duyne, Nano Lett., 2003, 3, 1057-1062.

182 L. J. Sherry, S.-H. Chang, G. C. Schatz and R. P. Van Duyne, Nano Lett., 2005, 5, 2034-2038.

183 W. J. Galush, S. A. Shelby, M. J. Mulvihill, A. Tao, P. Yang and J. T. Groves, Nano Lett., 2009, 9, 2077-2082.

184 D. E. Charles, D. Aherne, M. Gara, D. M. Ledwith, Y. K. Gun'ko, J. M. Kelly, W. J. Blau and M. E. BrennanFournet, ACS Nano, 2010, 4, 55-64.

185 L. J. Sherry, R. Jin, C. A. Mirkin, G. C. Schatz and R. P. Van Duyne, Nano Lett., 2006, 6, 2060-2065.

186 F. Tam, C. Moran and N. Halas, J. Phys. Chem. B, 2004, 108, 17290-17294.

187 N. Zhou, C. Ye, L. Polavarapu and Q. H. Xu, Nanoscale, 2015, 7, 9025-9032.

188 Y. Sun and Y. Xia, Anal. Chem., 2002, 74, 5297-5305.

189 H. Wang, D. W. Brandl, F. Le, P. Nordlander and N. J. Halas, Nano Lett., 2006, 6, 827-832.

190 G. Raschke, S. Brogl, A. S. Susha, A. L. Rogach, T. A. Klar, J. Feldmann, B. Fieres, N. Petkov, T. Bein, A. Nichtl and K. Kürzinger, Nano Lett., 2004, 4, 1853-1857. 